Engineering Technology Division

\title{
COUNTERCURRENT FLOW LIMITED (CCFL) HEAT FLUX IN THE HIGH FLUX ISOTOPE REACTOR (HFIR) FUEL ELEMENT
}

A. E. Ruggles

Date of Issue: October 12, 1990

\author{
Prepared for \\ Office of Energy Research \\ Basic Energy Sciences
}

\author{
Prepared by the \\ OAK RIDGE NATIONAL LABORATORY \\ Oak Ridge, Tennessee 37831 \\ operated by \\ MARTIN MARIETTA ENERGY SYSTEMS, INC. \\ for the \\ U.S. DEPARTMENT OF ENERGY \\ under Contract No. DE-AC05-84OR21400
}




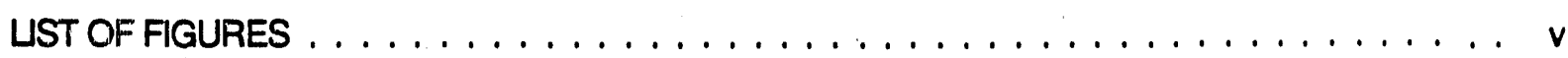

UST OF TABLES $\ldots \ldots \ldots \ldots \ldots \ldots \ldots \ldots \ldots \ldots \ldots \ldots \ldots \ldots$

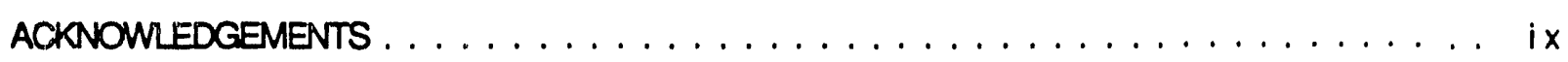

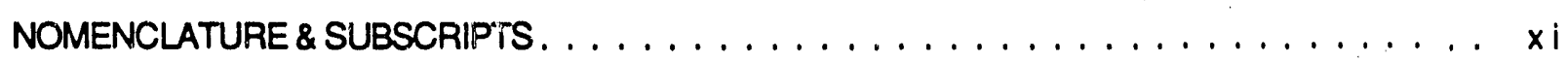

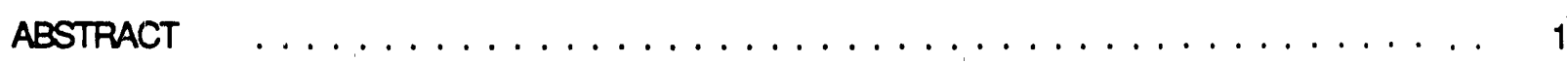

1. INTRODUCTION ........................... 1

2. ADIABATIC CCF INA SINGLE CHANNEL $\ldots \ldots \ldots \ldots \ldots$

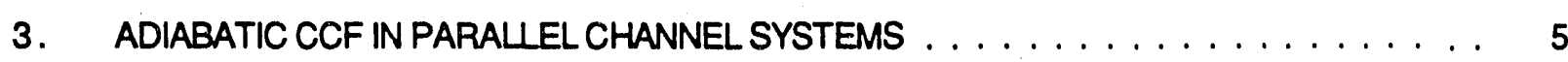

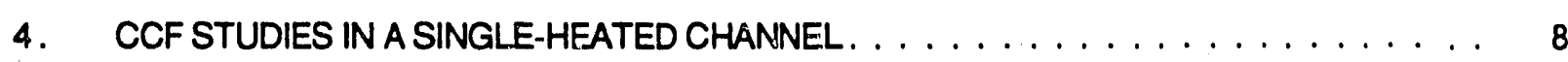

5. CCF STUDIES IN PARALEL HEATED TUBES $\ldots \ldots \ldots \ldots \ldots \ldots \ldots$

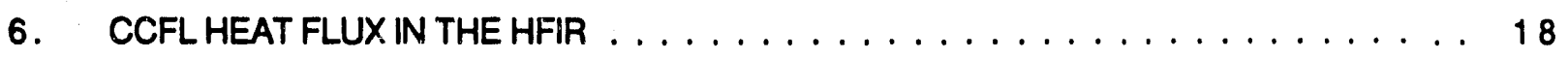

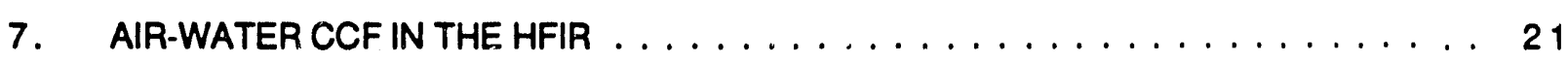

8. THE CCFL HEAT FLUXX PREDICTED BY THE MEASURED CCFL DATA . . . . . . . . 26

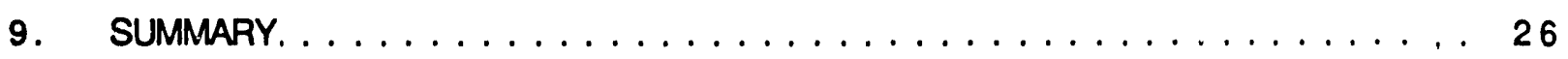

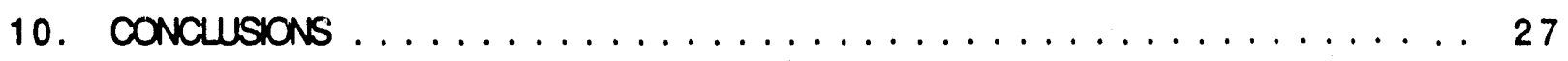

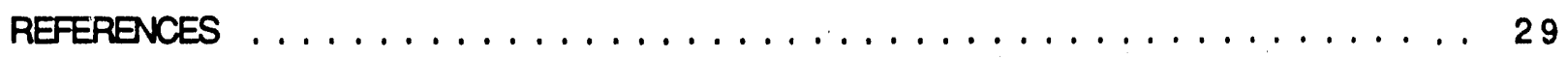

APPENDIX A: Mechanistic CCF Model (Fortran Listing) $\ldots \ldots \ldots \ldots$

APPENDIX B: Instrument Performance and Data Reduction Technique. . . . . . . . . . 37

APPENDIX C: Table C1: Penetration and Blowout Data . . . . . . . . . . 43

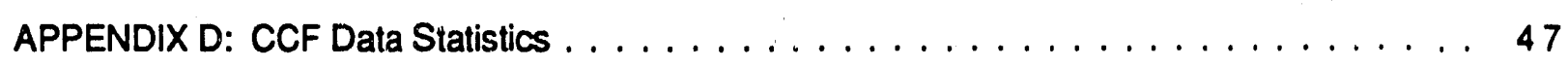

APPENDIX E: HFIR Fuel Plate Scoping Conduction Analysis . . . . . . . . . . . . . 51

$$
\text { iiifiv }
$$


Eia. No.

1 Test Section for Air-Water CCF Experiments (Mishima and Nishihara, 1984). . . 2

2 CCF Data Taken in Narrow Channels. . . . . . . . . . . . . . . . . 3

2a Data Due to Mishima and Nishihara, 1984 . . . . . . . . . . . . 3

2b Data Due to Osakabe and Kawasaki, $1989 \ldots \ldots \ldots \ldots \ldots$

3 Flow Geometry and Associated Characteristic Dimension for CCF Modeling Using the Wallis Correlation . . . . . . . . . . . . . . . . . . . . . . 5

4 Pressure Drop Characteristic for a Single Channel and Limiting Flow States for a System of Parallel Channels . . . . . . . . . . . . . . . . . . . 6

5 Countercurrent Performance for a System of Five Parallel Channels . . . . . . . . 7

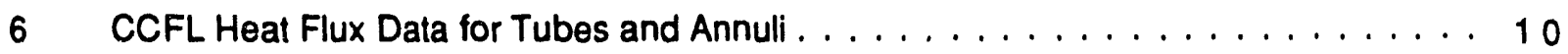

$7 \quad$ Local Pressure Drop of CCF in a Heated Tube $\ldots \ldots \ldots \ldots \ldots \ldots \ldots$

8 "Annular" Flow in a Narrow Channel . . . . . . . . . . . . . . . 13

9 Predicted CCF Performance as a Function of the Void Fraction. . . . . . . . . . 13

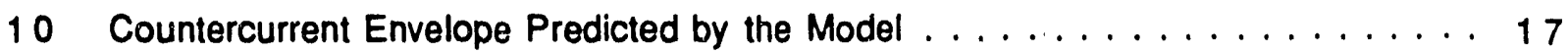

11 Schematic of the HFIR Pressure Vessel and Major Assemblies. . . . . . . . . . 18

12 HFIR Fuel Element Mock-Up Used as test Section . . . . . . . . . . . . . . . . 19

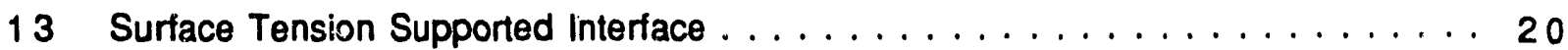

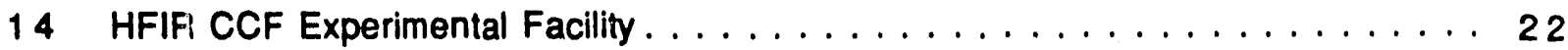

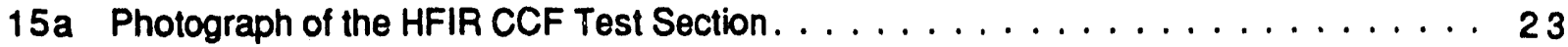

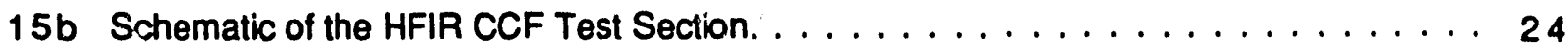

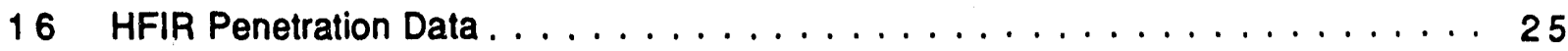

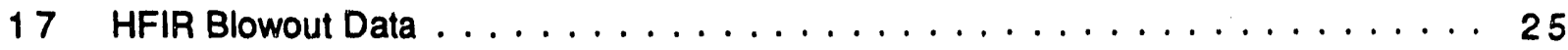


B.1 Calibration of Vortex Shedding Meter $\# 1 \ldots \ldots \ldots \ldots$

B.2 Calibration of Vortex Shedding Meter $\# 2 \ldots \ldots \ldots \ldots \ldots$

B.3 Calibration of Vortex Shedding Meter $\# 3 \ldots \ldots \ldots \ldots \ldots \ldots$

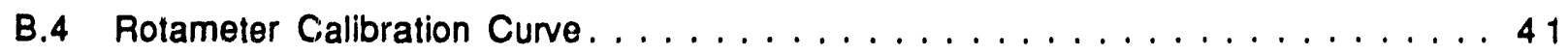

E.1 Fuel Plate and Associated Boundary Conditions for First Order Conduction Analysis . . 53 
Iable No.

1 Critical Heat Flux (CHF) Measurements for Blocked Downcomer Situations

(Gambill and Bundy, 1960) ....................... 9

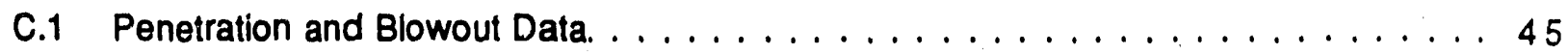

D.1 Statistics of the Linear Least Squares Fit to the Data . . . . . . . . . . . . . 49 


\section{ACHOWLEDGMENTS}

The detalled design of the experiment and instrumentation was performed by David Lannom at the K-25 Facility at Oak Ridge. Ed Biddle of the Engineering Technology Division of ORNL frequently operated the facility and took much of the reported data. 


\section{NOMENCLATURE}

$\begin{array}{ll}\text { A } & \text { area } \\ \text { b } & \text { intercept } \\ C_{w} & \text { Wallis constant } \\ D & \text { characteristic dimension } \\ g & \text { gravitational acceleration } \\ G & \text { mass flux } \\ j & \text { superficial velocity } \\ \text { K } & \text { conductivity } \\ \text { m } & \text { slope } \\ n & \text { span to gap ratio } \\ p & \text { pressure } \\ \Delta p & \text { Pinlet - Poutlet } \\ \text { S } & \text { channel span } \\ t & \text { fuel plate thickness } \\ w & \text { channel gap } \\ \rho & \text { density } \\ \Delta \rho & \text { PI- } \rho g \\ \sigma & \text { surface tension } \\ \lambda & \text { wave length }\end{array}$

SUBSCRIPTS

$\begin{array}{ll}\text { b } & \text { blowout line } \\ \text { CCF } & \text { counter-current flow limit } \\ \text { CL } & \text { centerline } \\ \text { G } & \text { gap } \\ \text { g } & \text { gas } \\ \text { HT } & \text { heat transfer } \\ \text { I } & \text { liquid } \\ \text { p } & \text { penetration line } \\ \text { sat } & \text { saturation } \\ \text { S } & \text { span } \\ \text { Xs } & \text { cross sectional } \\ \text { w } & \text { Wallis } \\ \text { A } & \text { position denoted on Figure } 5 \\ \text { B } & \text { position denoted on Figure } 5 \\ \text { fg } & \text { fluid to gas } \\ \text { C } & \text { characteristic } \\ \text { H } & \text { hydraslic } \\ \text { h } & \text { heaited } \\ \text { i } & \text { interfacial } \\ \text { S } & \text { span } \\ \text { T } & \text { Taylor }\end{array}$




\title{
COUNTERCURRENT FLOW LIMITED (CCFL) HEAT FLUX IN THE HIGH FLUX ISOTOPE REACTOR (HFIR) FUEL ELEMENT
}

\begin{abstract}
A. E. Ruggles
ABSTRACT

The countercurrent flow (CCF) performance in the fuel element region of the HFIR is examined experimentally and theoretically. The fuel element consists of iwo concentric annuli filled with aluminum clad fuel plates of $1.27 \mathrm{~mm}$ thickness separated by $1.27 \mathrm{~mm}$ flow channels. The plates are curved as they go radially outward to accomplish a constant flow channel width and constant metal-tocoolant ratio. A full-scale HFIR fuel element mock-up is studied in an adiabatic air-water CCF experiment. A review of CCF models for narrow channels is presented along with the treatment of CCFs in systems of parallel channels. The experimental results are related to the existing models and a mechanistic model for the "annular" CCF in a narrow channel is developed that captures the data trends well. The results of the experiment are used to calculate the CCFL heat flux of the HFIR fuel assembly. It was determined that the HFIR fuel assembly can reject $0.62 \mathrm{Mw}$ of thermal power in the CCFL situation.
\end{abstract}

\section{INTRODUCTION}

The HFIR is a plate-fueled research reactor that is normally cooled by the circulation of subcooled light water. The CCFL heat flux of the fuel element is important to the evaluation of the long-term decay heat removal capability of the reactor during certain hypothetical accident scenarios.

Many diabatic and adiabatic CCF experiments have been reported in the literature for tubes, annuli, and rod bundle geometries. Most of these efforts were motivated by decay heat removal concerns in power reactors after loss-of-coolant accidents. Only a limited number of CCF experiments have been conducted in narrow rectangular flow channels typical of a plate-fueled reactor. The limited amount of data, combined with the empiricism associated with existing CCF models, made a high confidence level calculation of the CCF performance in the HFIR fuel element impossible. Thus an adiabatic air-water CCF experiment was conducted using a full-scale HFIR fuel element mock-up to allow the unique core geometry to be fully represented and characterized.

Countercurrent flow behavior has been studied rather extensively. The reader is referred to Bankoff and Lee, 1983, for a critical review of the literature. Those issues pertinent to CCF in systems of parallel narrow rectangular channels is presented herein. This review begins by considering adiabatic CCF in a single channel and progresses to the more complicated situation of diabatic CCF in a system of parallel channels. 


\section{ADIABATIC CCF IN A SINGLE CHANNEL}

Adiabatic air-water CCF experiments have been performed on a single narrow rectangular channel by Mishima and Nishihara, 1984. Channel gaps of 1.5, 2.4, and $5.0 \mathrm{~mm}$ were examined. The channel width was $40.0 \mathrm{~mm}$ in all cases. The channel section, with associated entrance and exit geometries, is shown in Fig. 1. A similar set of experiments was performed by Osakabe and Kawasaki, 1989, where the channel span was $100 \mathrm{~mm}$ and channel gaps of 2.0, 5.0 , and $10.0 \mathrm{mIm}$ were tested. Both experiments were conducted using water at atmospheric pressure. The CCF data are shown in Figs. $2 a$ and $2 b$. The data are well represented by a correlation of the form

$\left[j_{g}^{\cdot}\right]^{1 / 2}+m\left[j_{i}^{\cdot}\right]^{1 / 2}=C_{w}$

ORNL-DWG 89.5183 ETD

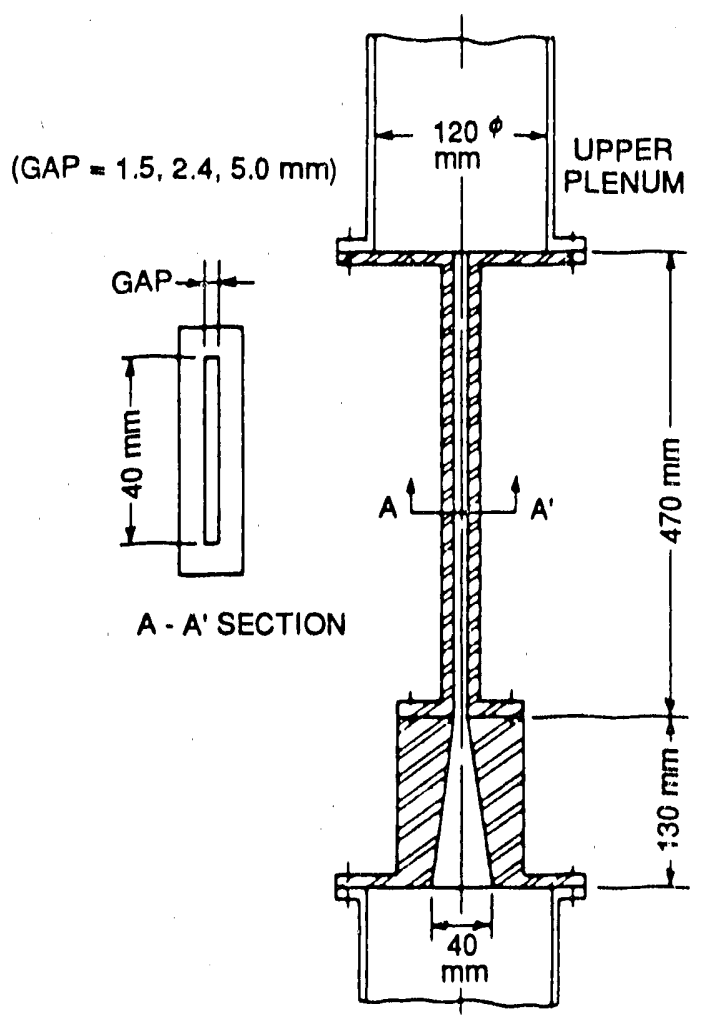

Fig. 1. Test Section for Air-Water Countercurrent Flow Experiments (Mishima and Nishihara, 1984) 


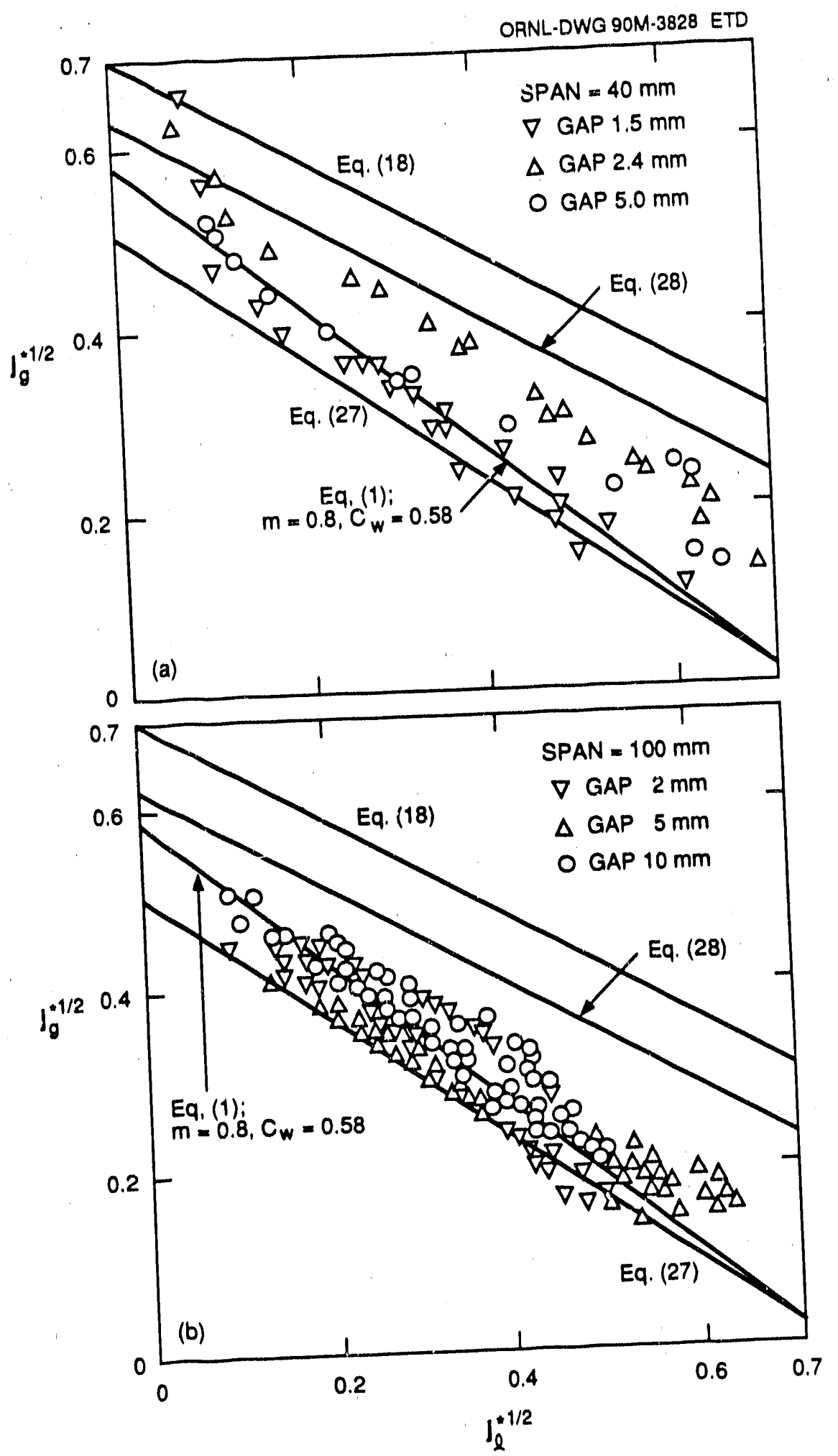

Fig. 2. Countercurrent Flow Data taken in Narrow Channels

a. Data due to Mishima and Nishihara, 1984

b. Data due to Osakabe and Kawasakl, 1989 
where the non-dimensional superficial velocities are defined using the channel span, $w$, as the characteristic dimension,

$$
\left[j_{g}\right]^{1 / 2}=\left[j_{g}\right]^{1 / 2}\left[\frac{\rho_{g}}{g[\Delta \rho] w}\right]^{1 / 4}
$$

and

$$
\left[j_{1}\right]^{1 / 2}=\left[j_{1}\right]^{1 / 2}\left[\frac{\rho_{1}}{g[\Delta \rho] w}\right]^{1 / 4} .
$$

Flow visualization studies by Mishima and Nishihara, 1984, and Osakabe and Kawasaki, 1989, indicate that most of the liquid downflow occurs at the ends of the channel span. Flow studies by Griffith, 1964, also show that the span influences the slip in two-phase flows in rectangular channels. These experimental observations help to support the use of the channel span dimension to nondimensionalize the superficial velocities when presenting CCF data. Osakabe and Kawasaki, 1989, found that values of $m$ equal to 0.8 and $C_{w}$ equal to 0.58 give the best fit to the data when the superficial velocity values were nondimensionalized in this manner, as shown in Figs. $2 a$ and $2 b$.

Figure 3 shows the range of characteristic dimensions that exist for various flow geometries that have been tested in CCF. Note that the annulus, which when rolled out resembles a narrow rectangular channel, is usually characterized by the hydraulic diameter, which is approximately two times the flow gap. However, situations exist where the circumference of the annulus is the appropriate dimension to use when modeling CCF behavior. No criterion exists to predict when to use the circumference to sharacterize iwo-phase CCF in an annulus. However, lannello and Todreas, 1989, have developed a model that predicts when countercurrent convective flows will occur somewhere around an annulus in single-phase systems. 

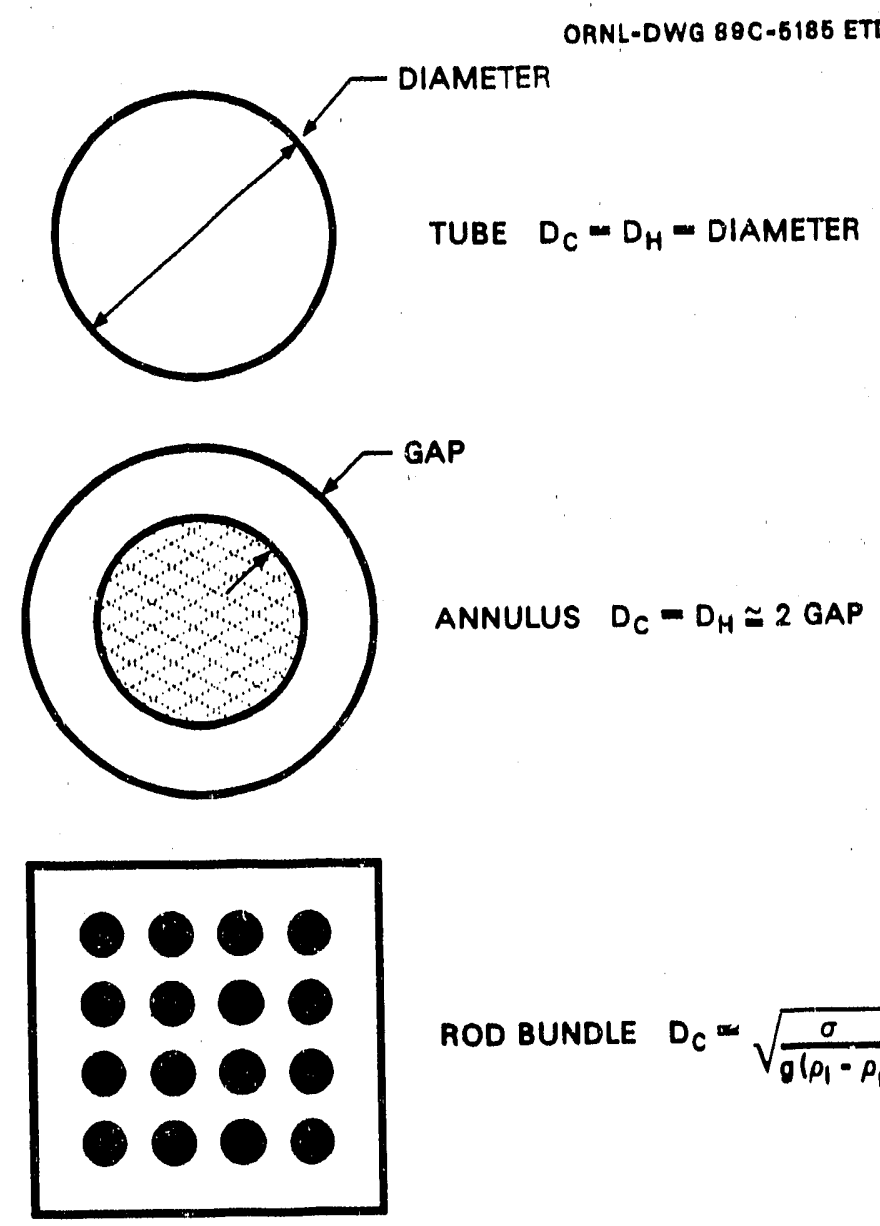

ROD BUNDLE $D_{C}=\sqrt{\frac{\sigma}{g\left(\rho_{1}-\rho_{\theta}\right)}}$

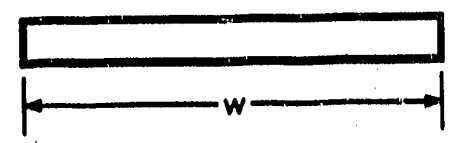

NARROW GHANNEL $D_{C}=2 W$

Fig. 3. Flow Geometry and Associated Characteristic Dimension for Countercurrent Flow Modeling Using the Wallis Correlation.

\section{ADIABATIC CCF IN PARALLEL CHANNEI SYSTEMS}

Wallis et al., 1981, developed an adiabatic CCF model for systems of parallel vertical tubes. The development of this model begins with the examination of the pressure drop characteristic of a single tube in the system as shown in Fig. 4. Note that there exists a range of pressure drop values between the so-called "penetration" and "blowout" lines where any one of four different flow situations can occur. CCF will only exist within this range of channel pressure drop. Fortunately, flow condition " $B$ " in Fig. 4 is not a stable condition for a single channel in a parallel channel system due to the Ledinegg, 1938, stability criterion, which predicts the 
ORNL-DWG 89C-5186R ETD

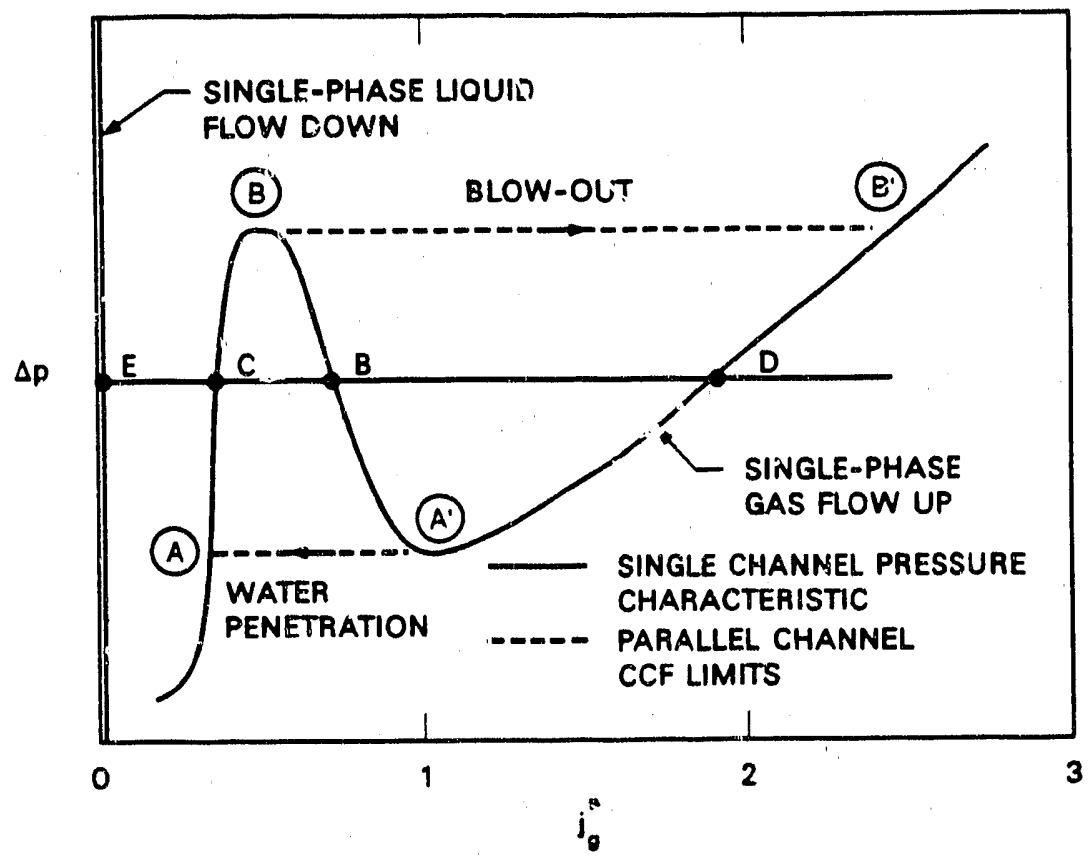

Fig. 4. Pressure Drop Characteristic for a Single Channel and Limiting Flow States for a System of Parallel Channels.

channel will be unstable when

$\frac{[d \Delta p]}{[d G]} \leq 0.0$

Thus, in practice, only one of three flow states may exist in a HFIR core channel during an airwater CCF experiment. This implies that for a system with $N$ parallel channels, $3 \mathrm{~N}-2$ different flow situations may exist for that system when the pressure drop is maintained somewhere between the hlowout and penetration values.

The CCF behavior of a single channel in the parallel channel situation can be obtained from the blowout and penetration characteristics of the parallel channel system. These characteristics are given for a system of parallel tubes in Fig. 5. The penetration characteristic is obtained by starting with all channels operating in single-phase gas upflow with water in the upper plenum. The gas flux is decreased until liquid penetration occurs. The system will then maintain a constant pressure drop equal to the penetration value as the gas flux is further decreased and more of the channels switch from single-phase upflow to two-phase CCF (see the dashed water penetration line in Fig. 4).

The blowout curve is obtained by starting with all channels in CCF and increasing the jas flux. This wiil take the sysiem upwaru ăiongy the iwio-pitase piessirie chaiacteristic showesn in Fig. 4 to 


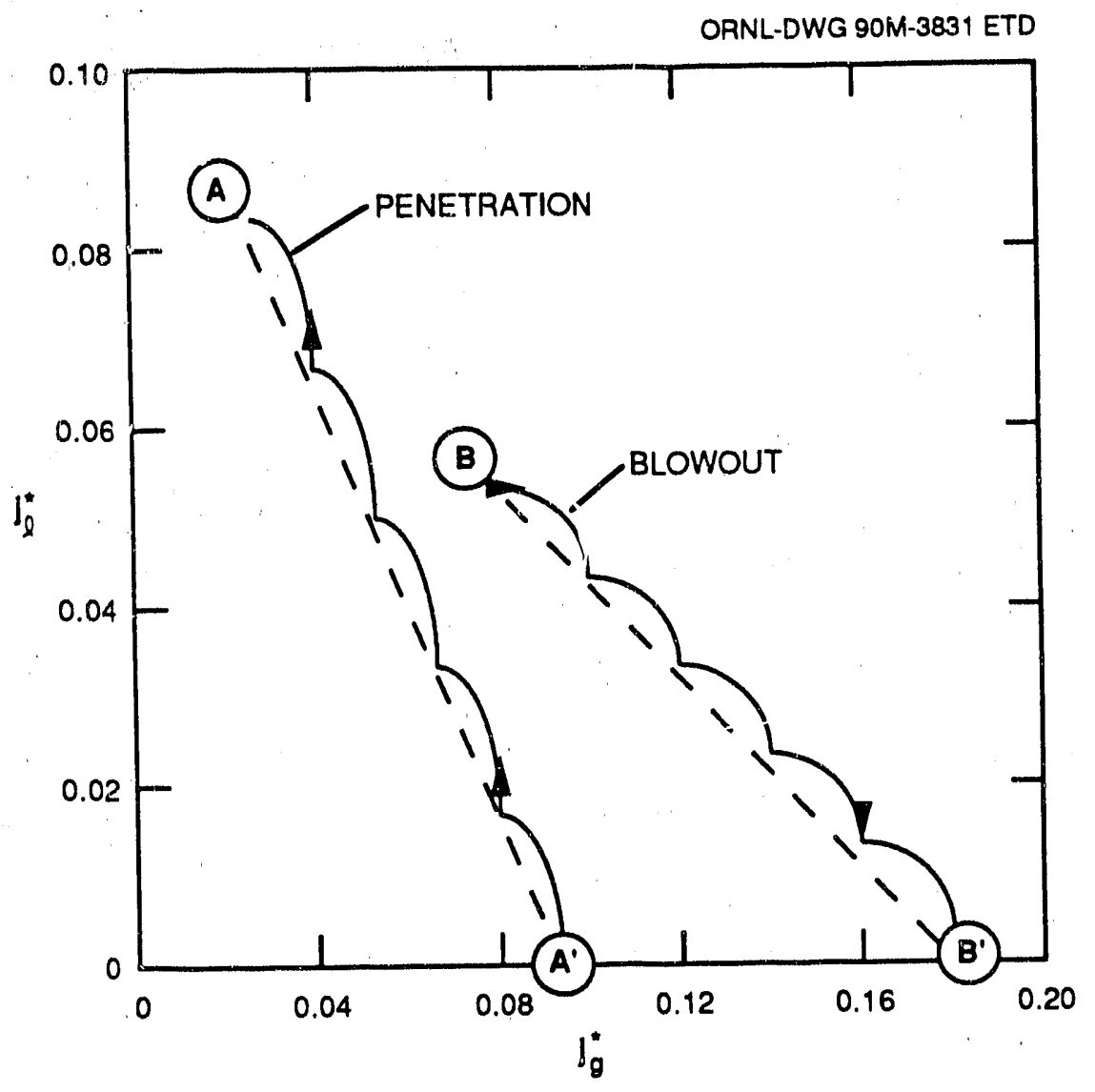

Fig. 5. Countercurrent Performance for a System or Five Parallel Channels

the blowout pressure drop value. Further increase in the gas flux will cause channels to switch to single-phase upflow while the system pressure drop remains constant.

The equations representing the system blowout and penetration characteristics were derived by Wallis et al., 1981, as

$j_{1}=B_{1}-B_{2} \dot{j}_{0}$

for penetration and

$j_{1}^{0}=B_{3}-B_{4} j_{0}^{\circ}$

for blowout. 
All the channels are in CCF at the far left of the penetration cunve, indicated by point $A$ in Fig. 5 . Thus the system of parallel channels has the superficial vapor velocity and superficial liquid velocity corresponding to CCF at the penetration pressure drop value at point $A$. All the channels are again in CCF at the far left of the blowe:: suive, indicated by point $B$. Thus the superficial vapor velocity and superficial :"quid velocity for the parallel channel system at point $B$ correspond to the CCF values at the blowout pressure drop. If the CCF behavior of the single channel in the parallel channel situation is assumed to take the form of Eq. (1), then the constants, $C_{w}$ and $m$, can be written as

$$
C_{w}=\frac{\left\{\left[j_{g, B}^{\cdot} j_{i, A}^{\cdot}\right]^{1 / 2}-\left[j_{g, A}^{\cdot j} j_{1 B}^{\cdot 1 / 2}\right\}\right.}{j_{i, A}^{1 / 2}-j_{i B}^{1 / 2}}
$$

and

$$
m=\frac{\left\{\left[j_{g, A}^{\cdot}\right]^{1 / 2}-\left[j_{g B}^{\cdot}\right]^{1 / 2}\right\}}{j_{i B}^{1 / 2}-j_{I, A}^{1 / 2}}
$$

where the superficial velocities are taken at points $A$ and $B$ indicated in Fig. 5. Note that all the channels are in C.CF during the tran. 'ion from point $A$ to point $B$ as shown in Figs. 4 and 5 . Therefore, these operating states may also be used to calculate $C_{w}$ and $m$.

\section{CCF STUDIES IN A SINGLE-HEATED CH,ANNEL}

The CCFL situation corresponds to when the mass flux of vapor upward equals tive mass flux of liquid downward,

$j_{g} \rho_{g}=j_{1} \rho_{1}$

The superficial f'is velocity at the CCF limit can be calculated from Eqs. (1), (2), and (7) as

$j_{g_{c o r}}=\left\{\frac{c_{w}}{\left[\frac{\rho_{g}}{g(\Delta \rho) 2 w}\right]^{1 / 4}+m\left[\frac{\rho_{v}}{\rho_{1}}\right]^{1 / 2}\left[\frac{\rho_{1}}{g(\Delta \rho) 2 w}\right]^{1 / 4}}\right\}^{2}$.

The CCFL heat flux is then calculated from an energy balance in conjunction with Eqs. $(7)$ and (8) as 
$q_{\text {core }}=\left[\frac{A_{x=}}{A_{H \pi}}-\left[\frac{C_{w}^{2} h_{1 g}\left(\rho_{g} g \Delta p w\right)^{1 / 2}}{\left[1+m\left(\rho_{g} / \rho_{1}\right)^{1 / 4}\right]^{2}}\right]\right.$

Mishima and Nishihara, 1985, conoucted CCFL Criticai Heat Flux (CHF) experiments in a narrow rectangular channel of dimensions $2.4 \mathrm{~mm} \times 40 \mathrm{~mm}$ with a $350 \mathrm{~mm}$ heated length. They found that Eq. (9) correlated their data well with $C_{w}=0.75$ and $m$ set equal to one. These values for $m$ and $C_{w}$ are almost identical to the values $m=1.0$ and $C_{w}=0.7 y$ derived from their adiabatic CCF tests (Mishima and Nishihara, 1984). Note that the values of $C_{w}$ presented here are larger than those published in the papers of Mishima and Nishihara by a factor of 2 to the $1 / 4$ power. This is due to the use of the channel span as the characteristic dimension in this paper while Mishima and Nishihara used two times the channel span in their publications.

Gambill and Bundy, 1960, performed a study of boiling natural circulation using a singleheated channel that included three measurements of CHF in a blocked downcomer situation. Table 1 shows these CHF values and their associated flow geometries. The values predicted by Eq. 9 using $C_{w}=0.75$ are also presented in Table 1.

Table 1. CHF Measurements for Blocked Downcomer Situations

(Gambill and Bundy, 1960).

\begin{tabular}{|c|c|c|c|c|}
\hline $\begin{array}{l}\text { Channel } \\
\text { Dimensions (mm) }\end{array}$ & $\begin{array}{l}\text { Heated } \\
\text { Lenath (m) }\end{array}$ & $\begin{array}{c}\text { Exit } \\
\text { Pressure (MPa) }\end{array}$ & $\begin{array}{l}\text { Measured Heat } \\
\text { Elux }\left(w / m^{2}\right)\end{array}$ & $\begin{array}{l}\text { Predicted Heat } \\
\left.\text { Elux (w/m } m^{2}\right)\end{array}$ \\
\hline $1.32 \times 28.2$ & 0.455 & 0.17 & 41,000 & 19,900 \\
\hline $1.32 \times 26.9$ & 0.455 & 0.17 & 62,100 & 19,600 \\
\hline $2.95 \times 52.6$ & 0.594 & 0.14 & 50,000 & 56,600 \\
\hline
\end{tabular}

No additional data involving narrow rectangular channels have been found to validate the performance of Eq. (9). However, a fairly large body of data taken in tubes and annuli exists to support this approach (Frea, 1970; Griffith et al., 1962; Kusuda and Imura, 1974; Mishima and Ishii, 1982; Barnard et al., 1973), as indicated in Fig. 6. Narrow channel CCFL data are included in Fig. 6 for comparison. The nondimensionalized axes used in Fig. 6 are defined as

$$
q^{*}=\frac{Q}{h_{1 g} A_{h}\left(\lambda \rho_{g} g \Delta \rho\right)^{1 / 2}}, \quad \text { where } \quad \lambda=\left(\frac{\sigma}{g \Delta \rho}\right)^{1 / 2}
$$

and

$$
\zeta=\frac{A_{x 8}\left(D^{\circ}\right)^{1 / 2}}{A_{h}\left(1+\left(\frac{P_{g}}{P_{1}}\right)^{1 / 4}\right)^{2}} \text {, where } \quad D^{\circ}=\frac{D_{c}}{\lambda} \text {. }
$$


ORNL-DWG 89-5188 ETD

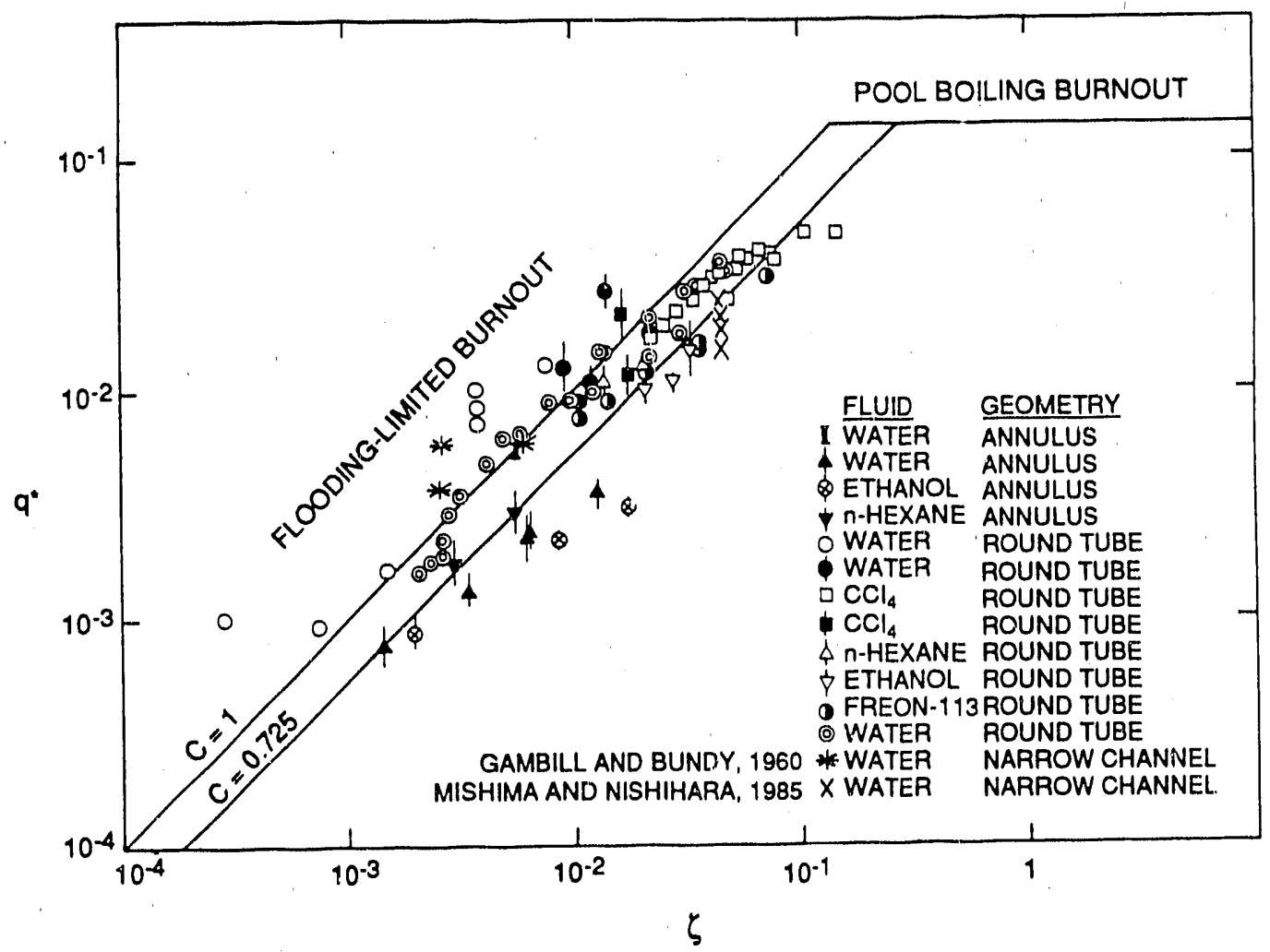

Fig. 6. Countercurrent Flow Limited Heat Flux Data for Tubes and Annuli

Note that the pool boiling departure from nucleate boiling heat flux value due to Zuber, 1959, is presented as the upper limit for a CCFL heat flux.

Mishima et al., 1985, found that the CHF at low mass flux is very sensitive to the amount of compressibility upstream of the heated section in the flow loop. The additici of upstream compressibility altered the amplitude and lengthened the period of the filow oscillations occurring in the heated channel. However, the CHF value in the blocked downcomer case was not observed to be sensitive to changes in the upstream compressibility in their experiments.

Some variation has been observed between CHF values measured in so-called blocked downcomer situations and those measured in blocked bottom situations. The heated channel is open to waterfilled plenums in the blocked downcomer situation. This allows for water to enter the channel from the top and bottom if significant compressibility exists in the plenums. Flow and pressure pulsations were observed in ihe tests performed by Gamble and Bundy, 1960, where two large thin-walled plenums were used. Some have suggested that the flow oscillations that can occur in the blocked downcomer case will increase the measured CHF value over that of the blocked bottom case where the bottom of the heated channel is closed to flow. This need not always be the case. Channels with low heat capacity are very vulnerable to so-called premature CHF during flow oscillations, especially if these oscillations are of low frequency. 
The blocked bottom situation and the blocked downcomer situation are the only two boundary conditions that have been tested for narrow channels. The boundary condition on a single-heated channel in the fuel element during the CCFL situation is one of constant pressure drop. No CCFL experiments have been conducted while holding such boundary conditions on a narrow rectangular heated channel.

\section{CCF STUDIES IN PARALLEL HEATED TUBES}

Many different two-phase flow situations may exist in a heated tube for a given pressure drop value as indicated in Fig. 7. Note that the heated tube may have both positive and negative mass flux situations resulting in the same pressure drop value. This type of behavior is possible due to the importance of buoyancy head in low mass flux two-phase flows and allows circulation loops to form within the parallel tube system as considered by Ostrogorsky et al., 1981, and Fakory and Lahey, 1983. The potential for these types of loops is enhanced if the power distribution varies among the channels in the system or if significant subcooling is available in the upper plenum. These circulation loops may exist even without net mass flux through the combined fuel assemblies. This behavior has been studied in the literature addressing "CCFL

ORNL-DWG 89C-6189ETD

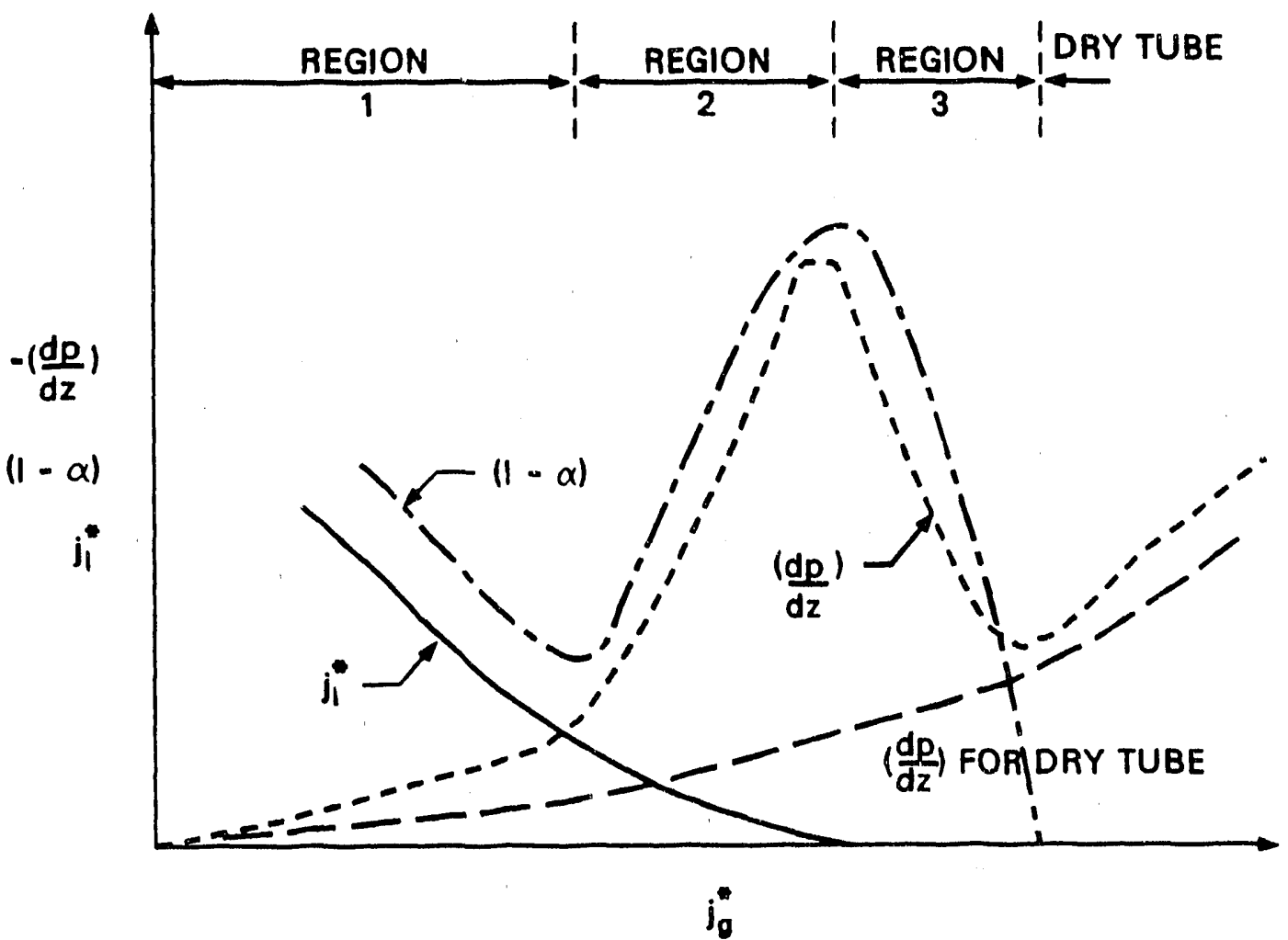

Fig. 7. Local Pressure Drop of Countercurrent Flow in a Heated Tube 
Breakciown" in conjunction with emergency core cooling system (ECCS) performance evaluations for power reactors.

The pressure drop characteristic of a CCF is usually modeled as having three regions as indicated in Fig. 7 (Ostrogorski et al., 1981, and Richter, 1981). The first region involves large values of liquid penetration and low values of gas flux. The interfacial shear is small compared to the wall shear. The pressure drop values are small since the wall shear and the gravitational forces acting on the liquid phase are nearly balanced (i.e., the liquid counterflow is essentially a falling film).

Interfacial shear and wall shear are both important in the second region. The addition of interfacial drag slows the liquid counterflow and thickens the liquid film. This region is characterized by increasing liquid volume fraction and a rough wavy liquid vapor interface. The channel pressure drop increases rapidly with increasing vapor flux.

A third region is encountered as the vapor flux is further increased and the liquid flux decreases. In this region the interface between the vapor and the liquid becomes more smooth and the pressure drop decreases with increasing vapor flux. The liquid film inickness decreases as the vapor flux is increased in this region.

All three of these situations may exist along a heated channel. Pressure drop relationships are well developed for tubes and annuli as presented by Ostrogorski et al., 1981, Wallis, 1969, and Richter, 1981. However, the CCF pressure drop in narrow channels is less well understood.

Mishima et al., 1984, developed a pressure drop model for the "annular" CCFs in narrow channels. This model assumed that the channel was dry along the span, contrary to the experimental observations. Thus the liquid iraction was partitioned equally between two films residing at each end of the channel span. This model did not compare well with their CCF data. The vapor velocities were over predicted as a result of assuming the channel walls were dry along the span, which resulted in an underrepresentation of the drag on the vapor core. No pressure drop data were available to test the model directly.

"Annular" CCF in narrow rectangular channels is unique in that two different liquid films exist as shown in Fig. 8. A model is developed herein that treats the two films separately. First, consider a force balance on a general one-dimensional film element as shown in Fig. 9:

$$
\frac{d p}{d z}+\rho_{1} g=\tau_{1} / \delta-\tau_{w} / \delta .
$$

An expression for the interfacial shear can be written as

$$
\tau_{1}=C_{1} \rho_{0}\left(u_{0}-u_{1}\right)^{2} / 2
$$

The drag coefficient, $C_{i}$, is taken from Wallis, 1969 , as

$$
C_{1}=C_{w}(1+300 \delta / D) \text {. }
$$


ORNL-DWG 89M-5191R ETD

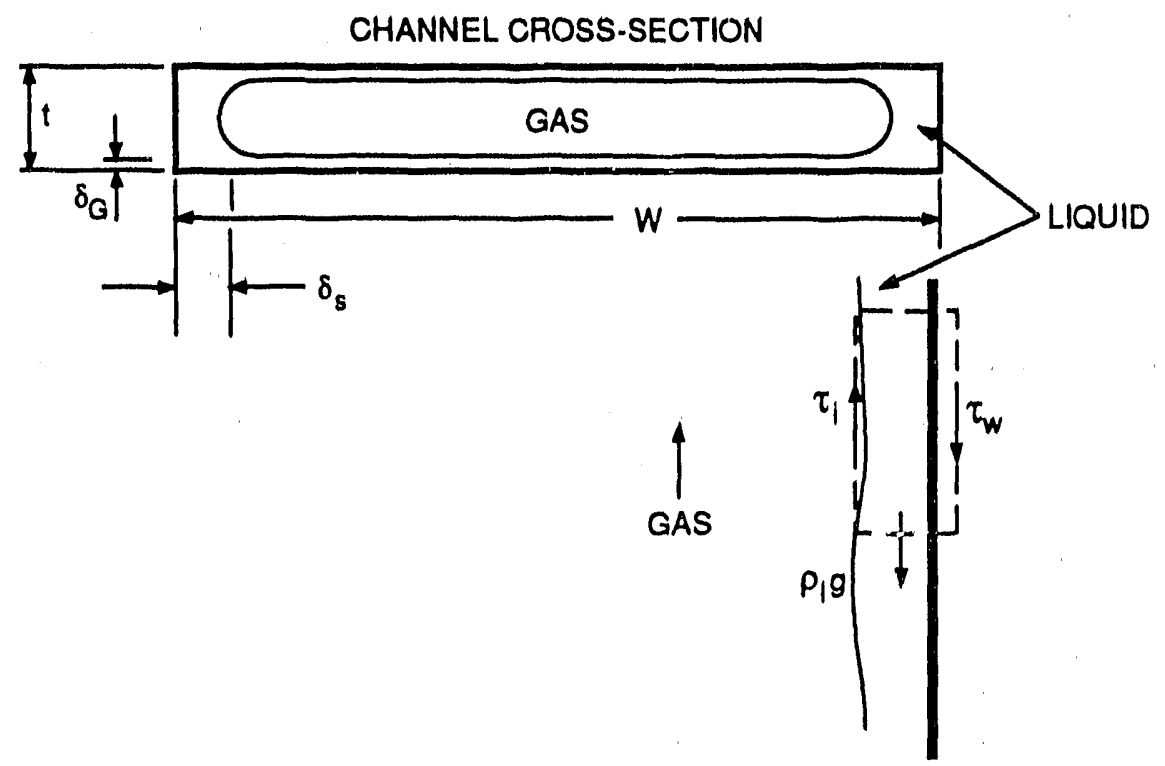

Fig. 8. "Annular" Flow in a Narrow Channel

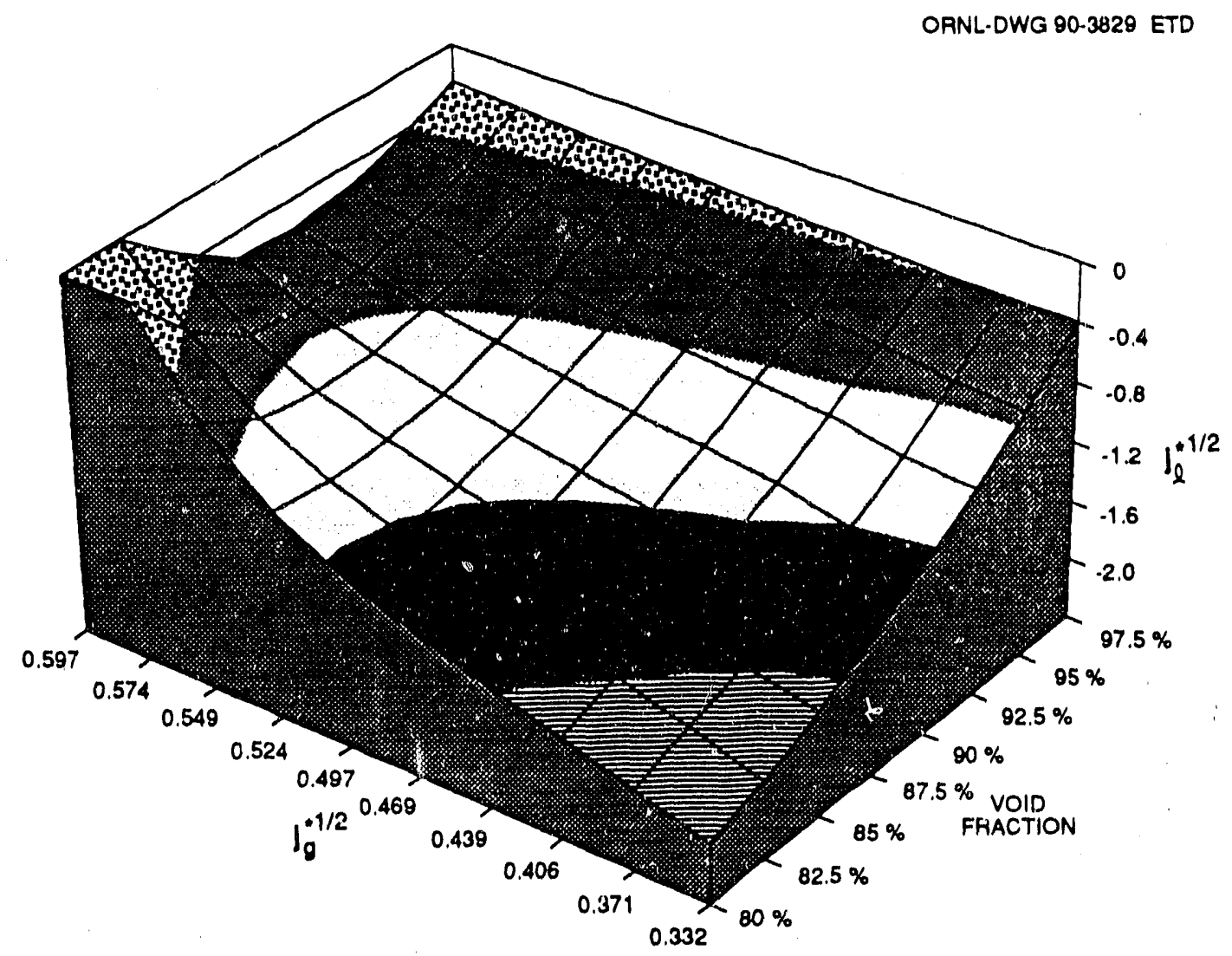

Fig. 9. Predicted Countercurrent Flow Performance as a Function of the Void Fraction. 
The value for the wall drag, $C_{\text {wall, }}$ is taken from Richter, 1981, as

$C_{\text {wall }}=0.008$

Note that the drag coefficient correlation described in Eqs. (14) and (15) was developed from tube data with the characteristic dimension of the tube taken as the diameter, $D$. These correlations will be employed for the film on the charriel walls using the channel gap as the characteristic dimension, as if an annular flow in a tube of diameter, $w$, were being considered. The channel span will be used as the characteristic dimension when considering the liquid film at the channel ends, as if an annular flow in a tube of diameter, $s$, was being considered.

It must be recognized that the gap-wise film may be ve $y$ thin when the channel gap is of the order of $1.0 \mathrm{~mm}$. Rivulet flows are presently excluded. ihis forces a defacto minimum film thickness of $0.01 \mathrm{~mm}$, as calculated from minimum surface energy considerations by Bankoff, 1971. The practical limit for this film thickness is probably somewhat larger.

The expression for the wall shear stress used by Richter, 1981, is given as

$$
\tau_{w, 1}=C_{w} \rho_{1} u_{1}\left|u_{1}\right| / 2
$$

A gravitational term derived from consideration of an assumed velocity gradient in the liquid film is sometimes included in the wall shear for annular flows (Ishii, 1977). This ierm is not used in this analysis. Richter's formulation is retained since it is consistent with the value of $\mathrm{C}_{\text {wall }}$ introduced in Eq. (15).

A force balance on the steam core yields

$$
\frac{d p}{d z}=\left[\frac{-\tau_{1, s} P_{1, s}}{\alpha A_{x s}}\right]-\left[\frac{\tau_{1, \sigma} P_{1, \sigma}}{\alpha A_{x S}}\right]-\rho_{g} g .
$$

A relationship between the interfacial shear stress terms in Eq. (17) is needed for closure. One way to postulate this relationship is to consider the velocity gradient in the vapor core, while continuing with the treatment of the "annular" narrow channel flow as if it were formed by two sets of "annular" flows between parallel plates. A fully developed turbulent velocity profile gives a friction factor for flow in a "fully rough" pipe that is independent of the Reynolds number (White, 1974). This implies that the pressure drop in an "annular" flow between parallel plates for a given mass flux will scale as the plate spacing. While this assumption is very approximate, it does formulate a limiting case for partitioning the pressure drop and interfacial shear stress, given by

$$
\tau_{1,8}=\tau_{1, \mathrm{~g}}\left[\frac{w}{s}\right] \text {. }
$$


This partitioning of the shear stress, when combined with Eq. (14), results it the film along the channel span carrying most of the liquid fraction:

$$
\alpha_{1, s}=\frac{-1+(w / s)}{150}+\alpha_{1, d}\left[\frac{w}{s}\right] .
$$

This is inconsistent with the experimentally observed flow distribution where much of the liquid was observed to be at the ends of the channel span.

Another situation can be postulated by assuming that the pressure drop in the vapor core is shared equally between the span. and gap-wise liquid films. The partitioning of the intertacial shear stress follows from Eq. (17) as

$$
\tau_{1,8}=\tau_{1, a}\left[\frac{s}{w}\right]
$$

Equation (20) combined with Eq. (14) produces the following partitioning of the liquid fraction:

$$
\alpha_{1,8}=\frac{-1+(s / w)}{150}+\alpha_{1, d}\left[\frac{s}{w}\right] .
$$

This case indicates that no liquid will be on the channel walls until the liquid fraction at the ends of the channel span exceeds a minimum value given by

$$
\alpha_{1,0}=\frac{(s / w)-1}{150}
$$

Thus a channel ivith a spari-to-gap ratio of thinty will not have liquid on the channel walls (l.e., a gap-wise liquid film) until the total liquid volume fraction approaches $20 \%$. This is the assumption made tacitly by Mishima and Nishihara, 1984, when they modeled the channel span as dry and simply used a single-phase friction factor. Their model did not perform well, and the channel walls were not observed to be dry, even though materials with very poor wetability were used.

Two extreme cases have been considered, one where the interfacial shear due to the span-wise film is much less than the interfacial shear due to the gap-wise film. and one where the interfacial shear due to the span-wise film is much greater than the interfacial shear due to the gap-wise film. Neither of these assumed cases predict the observed phase distribution in the channel. A third case can be developed by assuming the interfacial shear stress is the same for the film along the channel span as for the film across the channel gap,

$$
\tau_{1, s}=\tau_{1,0}
$$


This assumption is roughly consistent with assuming that the velocity distribution in the vapor core is uniform across the span. The distribution of liquid follows from Eqs. (13), (14), and (23) as

$\delta_{s} / s=\delta_{G} / w$.

Thus the total liquid fraction of the flow is given by,

$$
\alpha_{1}=4 \delta_{s} / s=4 \delta_{G} / W \text {. }
$$

Equations (24) and (25) together indicate that $50 \%$ of the liquid fraction will lie in the films along the channel span, and $50 \%$ of the liquid fraction will lie in the films across the channel gap, regardless of the ratio of span to gap.

Equations (12) through (20) can be combined to produce separate force balances for the gapand span-wise liquid films, respectively,

$$
\left(\rho_{1}-\rho_{g}\right) g=\tau_{1}\left\{v \delta_{G}+P_{1} / \alpha A_{x S}\right\}-\left\{C_{w a \mid 1} \rho_{1} u_{1, Q}\left|u_{1, Q}\right| 2\right\} V \delta_{G}
$$

and

$$
\left(\rho_{1}-\rho_{g}\right) g=\tau_{1}\left\{v \delta_{s}+P_{1} / \alpha A_{x s}\right\}-\left\{C_{w a l l} \rho_{1} u_{1, s}\left|u_{1 s}\right| v 2\right\} V \delta_{s} .
$$

The interfacial perimeter, $P_{l}$, is taken as

$$
P_{1}=P_{w}\left(1-\alpha_{1} / 2\right)
$$

where $P_{w}$ is the wetted perimeter of the channel.

Equations (26), (27), and (28) can be combined with Eqs. (13), (14), (15), and (25) and solved in favor of the liquid velocities, $u_{,, G}$ and $u_{I, s}$, respectively,

$$
\begin{aligned}
& u_{1, G \mid}\left|u_{1, Q}\right|=\left(D_{Q} \alpha_{1} /\left(4 \rho_{j}\right)\right)\left(1+75 \alpha_{1}\right) \rho_{o}\left(u_{0}-u_{1}\right)^{2}\left\{4 / D_{Q} \alpha_{1}+\left(1-\alpha_{1} / 2\right) P_{w} / \alpha_{g} A_{x=}\right\} \\
& -\left(\rho_{1}-\rho_{g}\right) g D_{Q} \alpha_{1} /\left(2 C_{p_{1}}\right) \text {, } \\
& u_{1 s}\left|u_{1 s}\right|=\left(D_{s} \alpha_{1} /\left(4 \rho_{1}\right)\right)\left(1+75 \alpha_{1}\right) \rho_{g}\left(u_{g}-u_{1}\right)^{2}\left\{4 / D_{s} \alpha_{1}+\left(1-\alpha_{1} / 2\right) P_{w} / \alpha_{g} A_{x_{8}}\right\} \\
& -\left(\rho_{1}-\rho_{g}\right) g D_{s} \alpha_{1} /\left(2 C_{1} \rho_{1}\right) .
\end{aligned}
$$


The total average liquid superficlal velocity in the channel is given by

$j_{1}=\left[u_{1,0}+u_{1, s}\right] \alpha_{1} / 2$

Equations (29) through (31) can be solved to produce the CCF surface shown in Fig. 9. The velocity of the liquid at the interface, $u_{i}$, is assumed to be small relative to the gas velocity consistent with the development due to Richter, 1981. The superficial gas velocity and the superficial liquid velocity are thus uniquely related for each chosen value of the channel average void fraction. The flow channel cross section used to create Fig. 9 is $1.5 \mathrm{~mm}$ by $40 \mathrm{~mm}$, which is consistent with one of the flow channel cross sections tested by Mishima and Nishihara, 1984. The Fortran program used to produce Fig. 9 is documented in Appendix A.

The locus of CCF curves can be plotted in a manner similar to that employed by Mishima et al., 1984, and Ohkawa and Lahey, 1980, as shown in Fig. 10. The right boundary in Fig. 10 gives the CCF performance of the narrow channel as predicted by the mechanistic model. ithis boundary can be approximated by an equation in the form of Eq. 1 where $m$ is equal to 0.55 and $C_{w}$ is equal to 0.70 . This result is plotted with the CCF data from Mishima ef al., 1984, and Osakabe and Kawasaki, 1989, in Fig. 2.

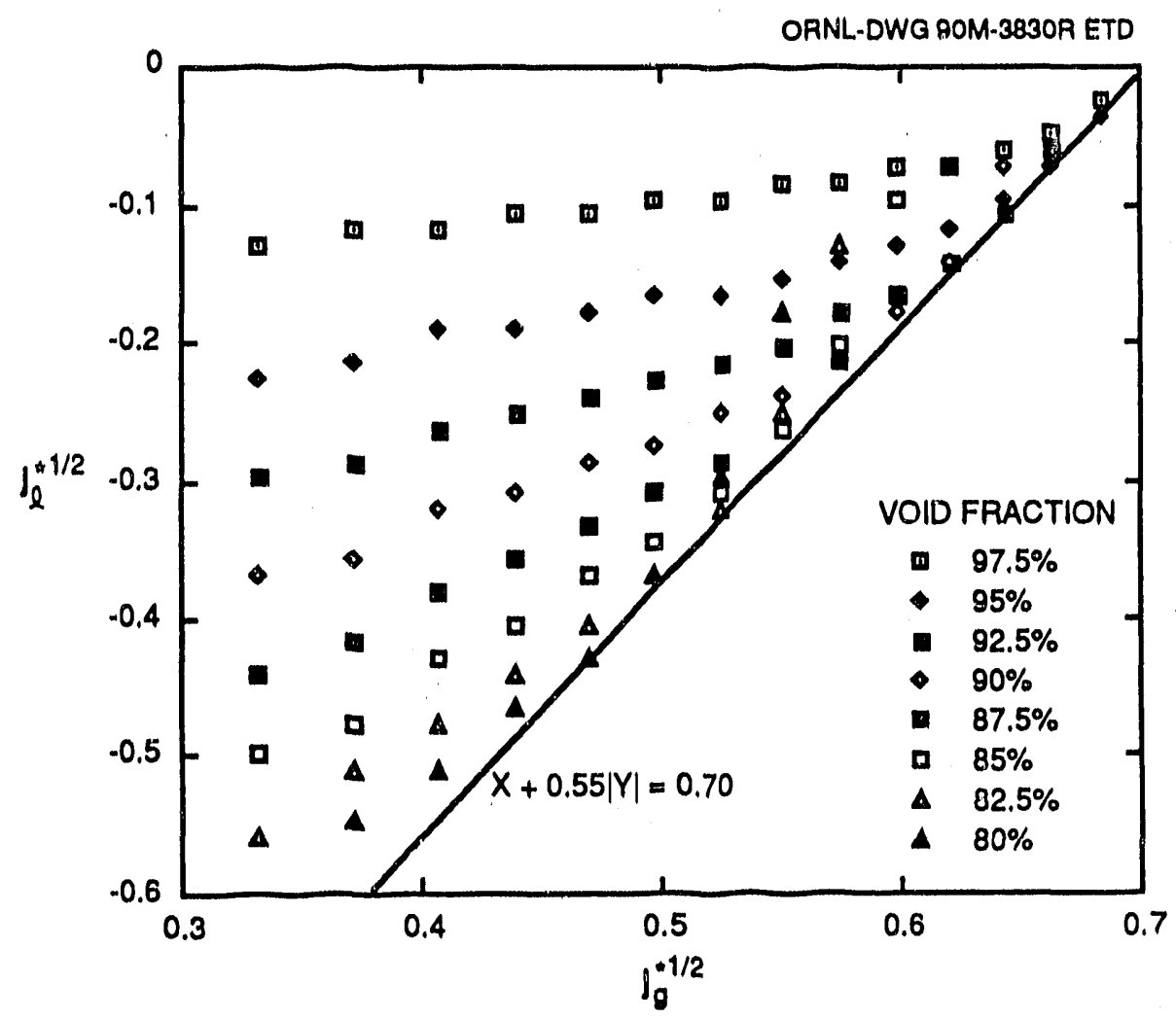

Fig. 10. Countercurrent Envelope Predicted by the Model 
Note that the momentum balance developed in this section is only appropriate to high void fraction annular flows with wavy interfacial boundaries. The model predicts that most of the liquid downflow is due to the thicker films at the ends of the channel span. The thinner films along the channel span contribute most of the drag on the vapor core but are responsible for very little liquid downflow. This behavior is consistent with the reported flow observations.

\section{CCFL HEAT FLUX IN THE HFIR}

The circumstances being considered for the HFIR during decay heat removal are quite different from those generally postulated during ECCS operation in a power reactor. The HFIR fuel element is submerged, as shown in Fig. 11, with water avallable both below and above the fuel

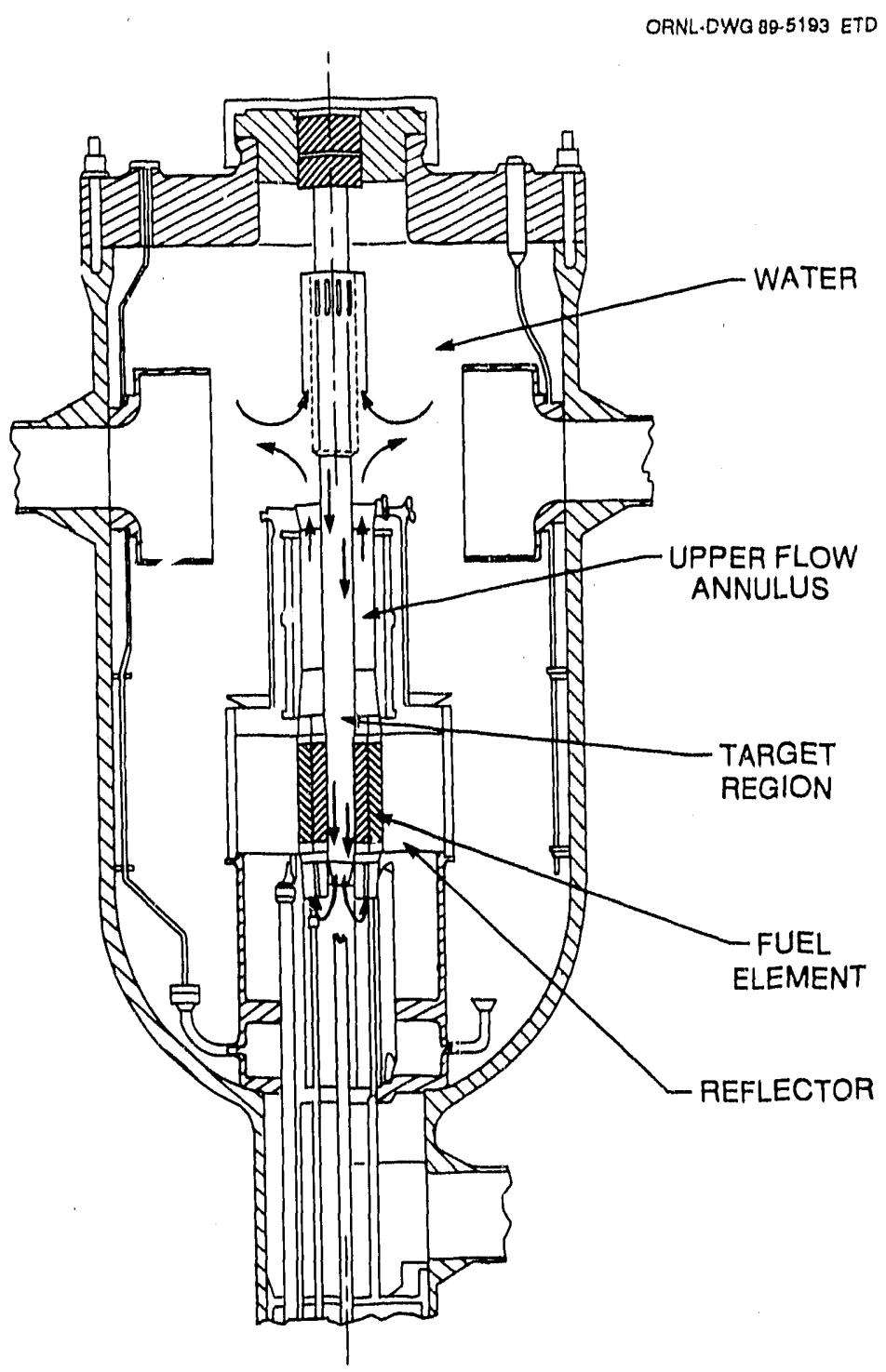

Fig. 11. Schematic of the HFIR Pressure Vessel and Major Assemblies 
element. The core region is near atmospheric pressure. The decay heat in the fuel element causes a natural circulation flow within the pressure vessel. The exact amount of mass flux upward through the fuel element due to natural circulation is difficult to determine. Therefore, the mass flux of liquid entering the bottom of the fuel element has been assumed to be zero. CCF at the top of the fuel element will establish the heat flux that the fuel element can reject under these circumstances. The heat flux corresponding to the CCFL situation is less than that when a net upward mass flux is allowed through the channel (Mishima and Ishil, 1982). Note that no possibility exists for single-phase vapor upflow in some channels, as might occur in a power reactor during ECCS operation when vapor is avallable biolow the fuel assemblies.

The potential for single-phase or low void fraction two-phase downflows does exist if the upper flow annulus water has substantial subcooling. However, the water in the upper flow annulus will generally be near saturation since a low mass flux two-phase boiling natural circulation flow is passing through the fuel element. Again, this departs from the situation postulated during ECCS operatior, in a power reactor where the EC.CS water may be highly subcooled.

The dimensions of the HFIR fuel element are given in Fig. 12. The flow passages are $1.27 \mathrm{~mm}$ in gap and are spaced by $1.27 \mathrm{~mm}$ fuel plates. The close proximity of the flow channels to one

ORNL.DWO 89.3996 ETD

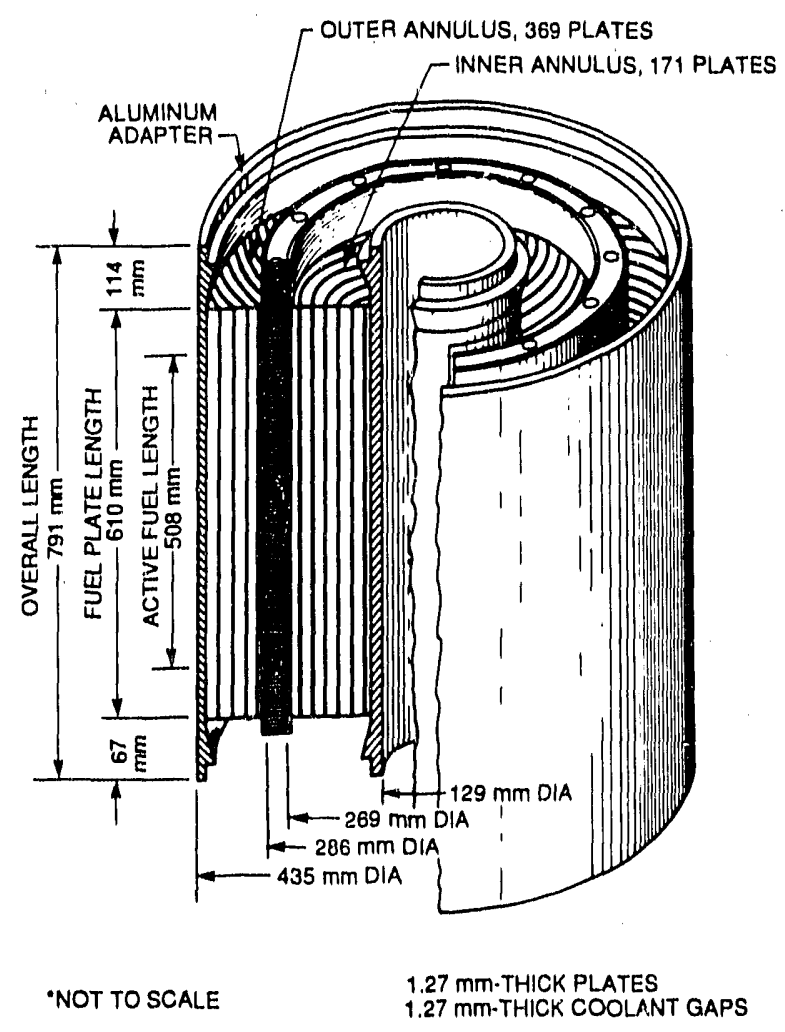

Fig. 12. HFIR Fuel Element Mock-Up Used as Test Section 
another is quite different from the situation tested in other parallel channel CCF experiments (Wallis et al., 1981, Suzuki et al., 1988, Fakory and Lahey, 1983, Thomas and Combs, 1983). It is well known that the geometry of the channel exit (i.e., at the channel top) can have a significant impact on the CCF behavior. It is interesting to note that in this case the spacing of the channel exits, $2.54 \mathrm{~mm}$, is less than half of a Taylor wavelength, $\lambda_{T} / 2=8.0 \mathrm{~mm}$, as given by

$$
\lambda_{T} / 2=\pi\left[\frac{\sigma}{g\left(\rho_{1}-\rho_{g}\right)}\right]^{1 / 2} .
$$

This indicates that surface tension effects may be important to the flow behavior at the intersection of the fuel element and the upper flow annulus. A photograph of an air/water interface created by inverting a small glass of tap water onto a grid of nylon filaments is given in Fig. 13. The spacing of the nylon filaments connecting the surface tension supported liquid 'droops' is approximately identical to the HFIR fuel plate spacing. A similar interface is not expected at the intersection of the fuel elerrant and upper flow annulus. However, some restriction of the liquid flow into the channel along the span may result from surface iension effects. It is interesting to note that the spacing of vapor jets used in the mechanistic pool boiling critical heat flux model due to Zuber, 1959 , is approximately equal to $\lambda_{T} / 5$.

ORNL-DWG $90-3934$ ETD

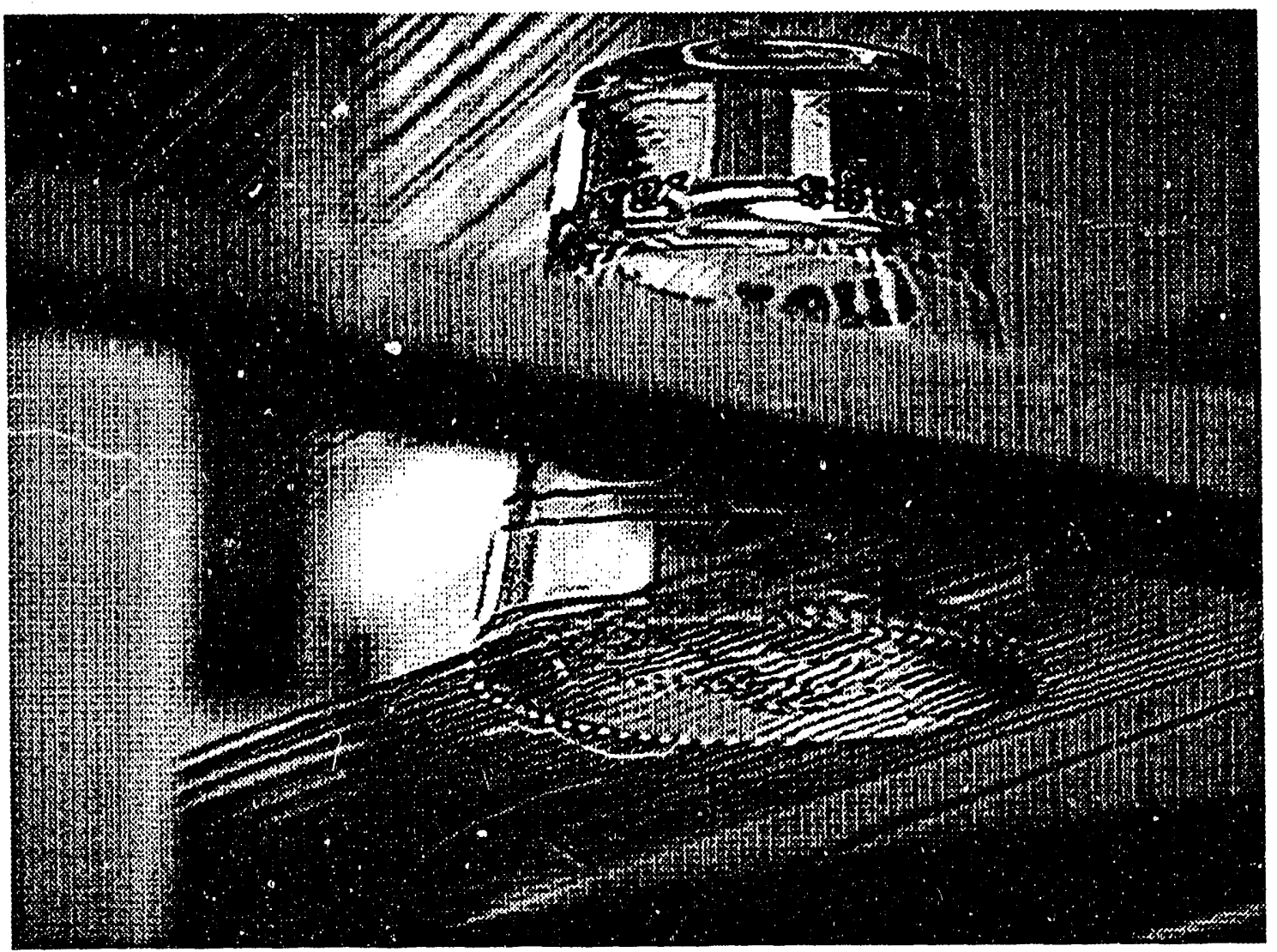

Fig. 13. Surface Tension Supported Interface. 
The CCFL heai flux predicted by Eq. 9 goes as the channel span dimension to the $1 / 2$ power. This relationship has only been validated for channel span values up to $40 \mathrm{~mm}$. The HFIR channel span is $84 \mathrm{~mm}$. The CCFL heat fiux predicted by Eq. (9) goes to infinity as the channel span value goes to infinity. Clearly the channel gap will limit the CCFL heat flux to soine finite value. The range of channel gap values (or span-to-gap ratios) over which Eq. (9) may be applied is yet to be determined.

A full scale CCF experiment was conducted using a HFIR fuel element mock-up in order to arrive at a defendable model for the CCF performance of the HFIR in a timely manner. This approach was further expedited since metered air and water supplies were already available that could accomplish a full-scale CCF experiment. Unfortunately, separate effects studies of the individual phenomena imbedded in the full-scale experiment were not possible.

\section{AIR-WATER CCF IN THE HFIR}

Several penetration and blowout characteristics of the type shown in Fig. 5 were measured using a HFIR core mock-up in a CCF loop. A schematic of the flow loop and associated instrumentation is given in Fig. 14. A photograph and schematic of the test section, including the HFIR fuel element mock-up and the upper and lower plenums are given in Figs. 15a and 15b. The fuel element mock-up is as shown in Fig. 12. The outermost and innermost boundary lubes of the fuel element are extended $0.33 \mathrm{~m}$ above the fuel element to simulate the upper flow annulus. Calibration curves for the air flow meters and water flow meter are given in Appendix $B$ along with the technique for temperature and pressure compensation of the measured air flows.

The liquid penetration data were measured starting with a gas flux that would not allow liquid penotration through the fuel element. The upper plenum was then filled with water to a predetermined level while the gas flux was maintained through the fuel element. The gas flux was then slowly decreased until liquid penetration occurred. The gas flux was further decreased, and the corresponding countercurrent liquid flux values were measured. The measured penetration curve is given 'n Fig. 16.

The liquid blowout data were measured starting at the end of a set of liquid penetration measurements. The gas flux was increased, and the corresponding values for the liquid flux were recorded. This process was continued until the liquid flux values were too small for accurate measurement. The measured countercurrent blowout data is given in Fig. 17. Only data taken after a near constant pressure drop value was established were admitted. This is consistent with the theory presented for adiabatic CCF in parallel channel systems since points on the pressure drop characteristic (see Fig. 4) leading upward from the penetration value to the blowout value are not part of the blowout curve.

Least squares linear fits to the data are shown in Figs. 16 and 17. All the data were taken at near atmospheric conditions with water and air temperatures averaging $27^{\circ} \mathrm{C}$. The data are tabulated in Appendix C. 
ORNL-DWG 89-5194 ETD

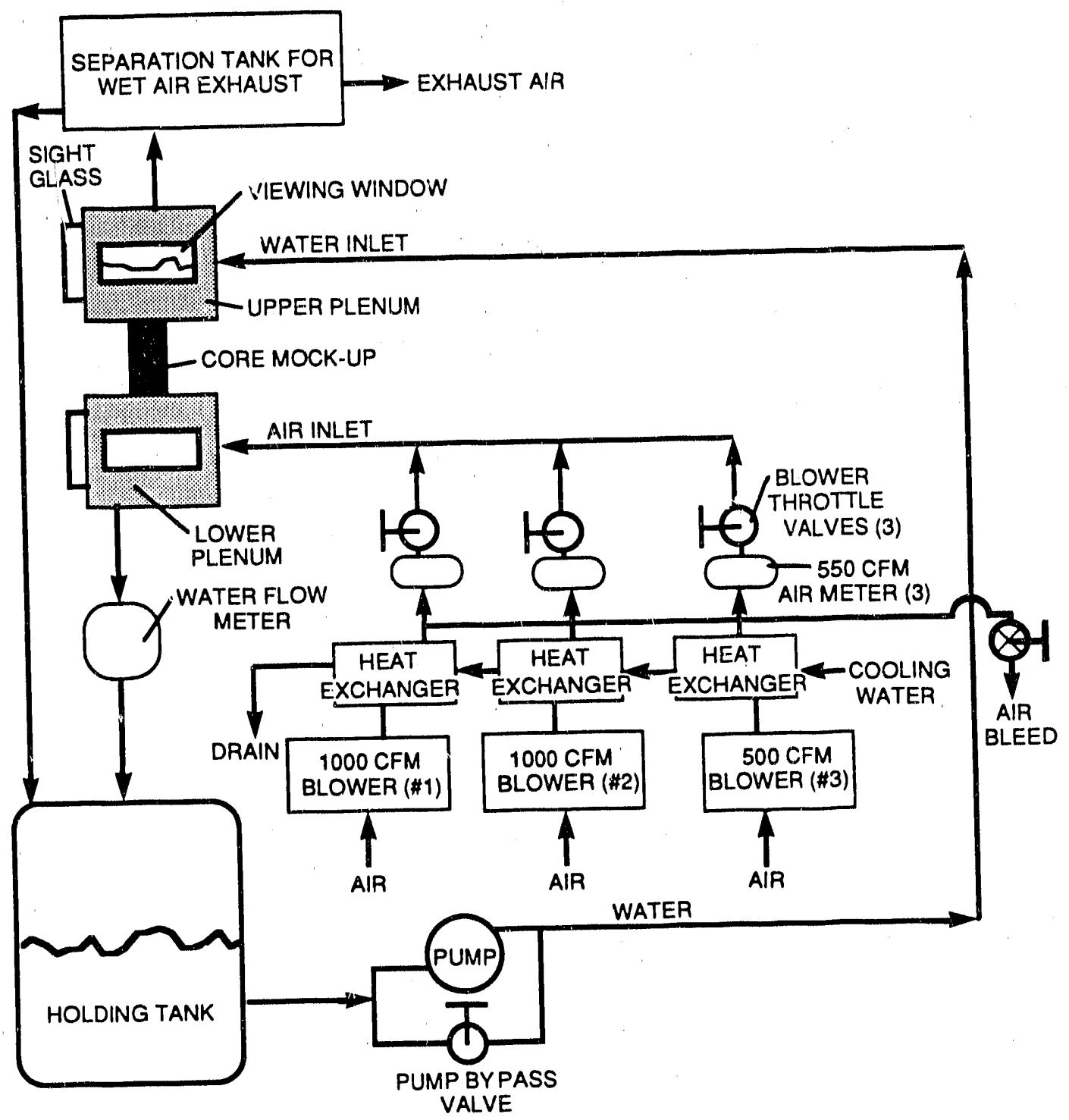

Fig. 14. HFIR CCF Experimental Facility

The linear representations of the penetration and blowout data are nondimensionalized consistent with Eq. (2) and are in the form of Eqs. (4) and (5), with

$j_{1}^{*}=0.18-1.04\left[j_{g}^{*}\right]$

for the penetration characteristic and

$j_{1}=0.13-0.63\left[i_{0}^{\circ}\right]$ 


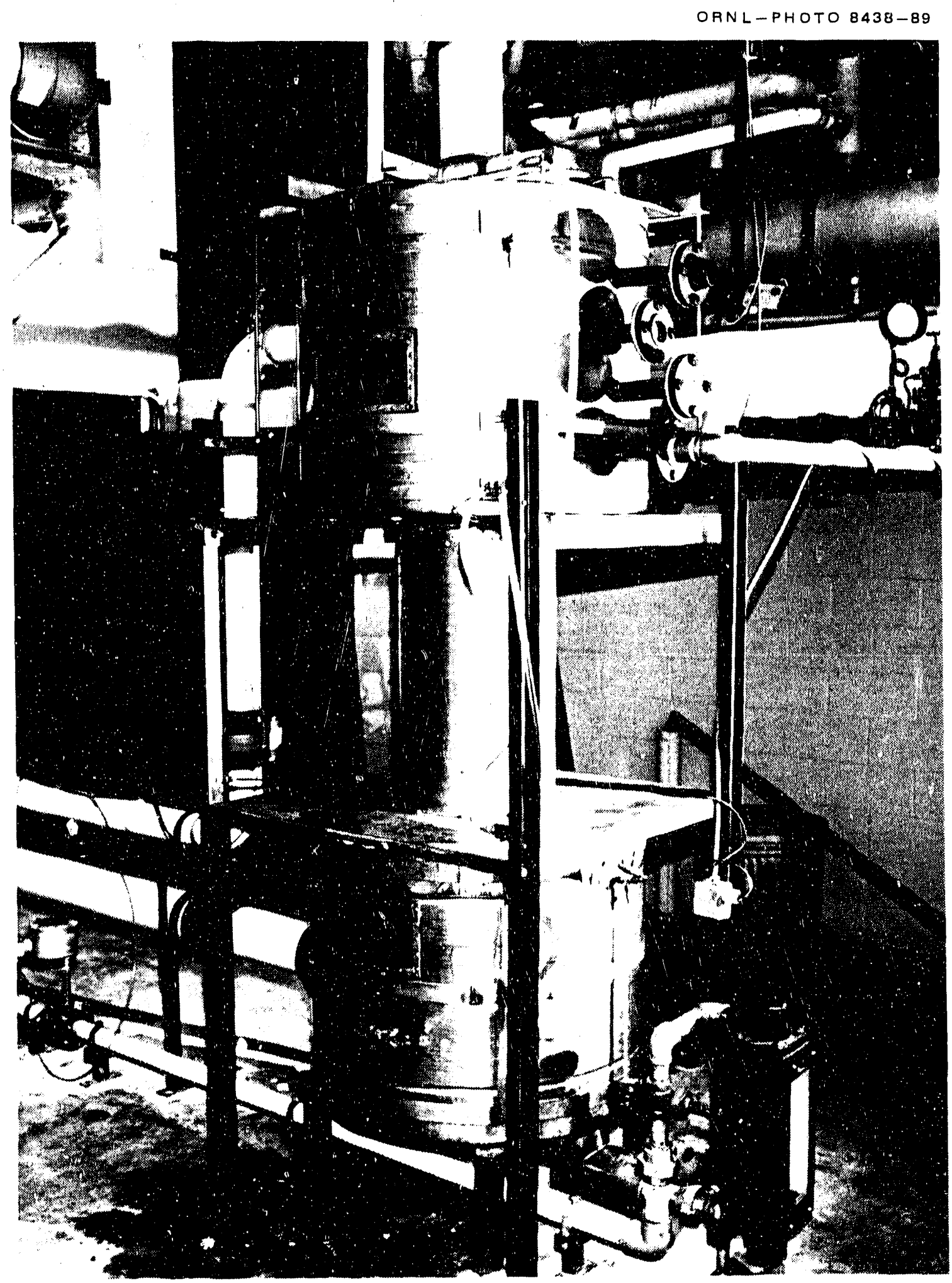

Fig. 15a. Photograph of the HFIR CCF Test Section 
ORNL-DWG 89-5195 ETD

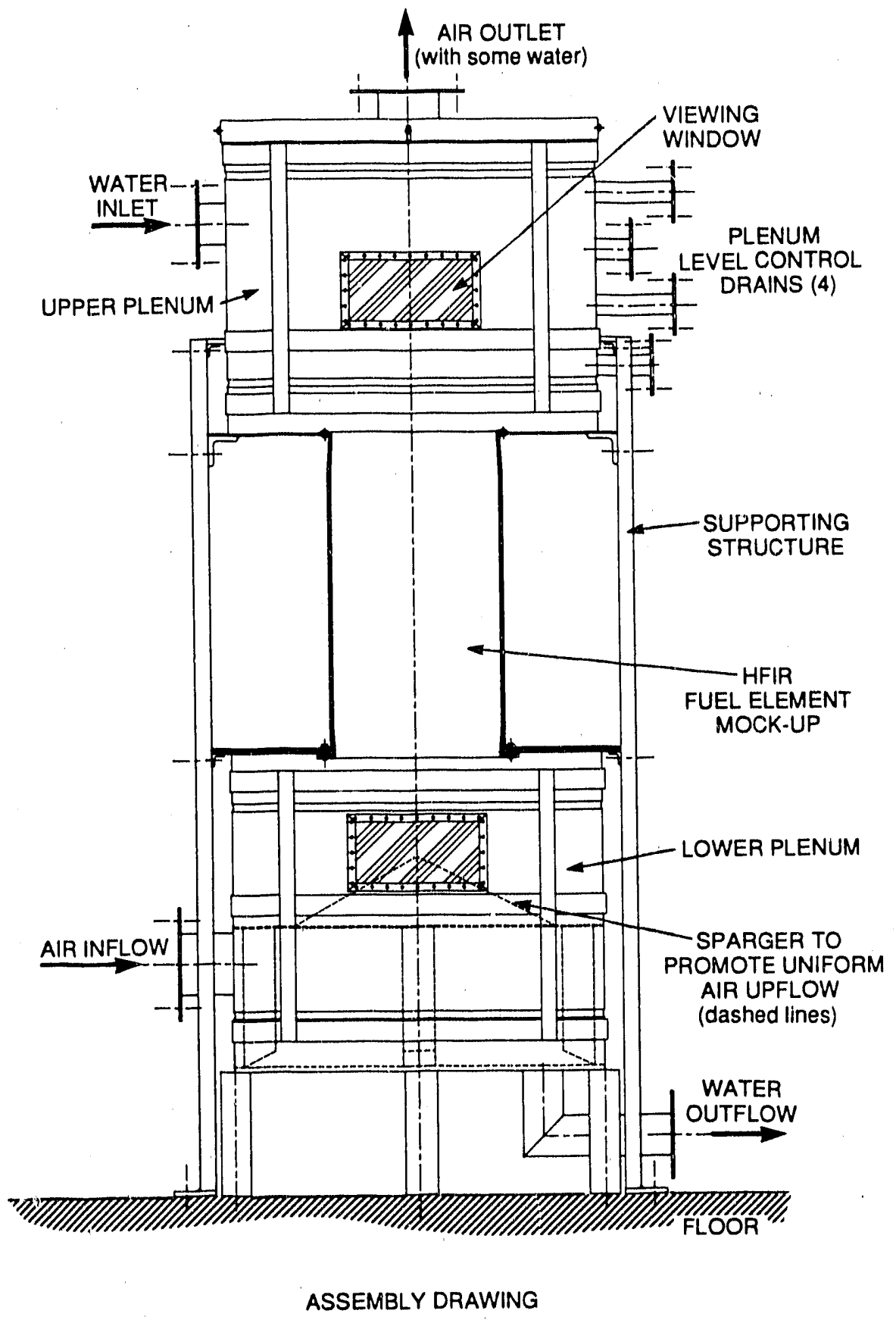

Fig. 15b. Schematic of the HFIR CCF Test Section 


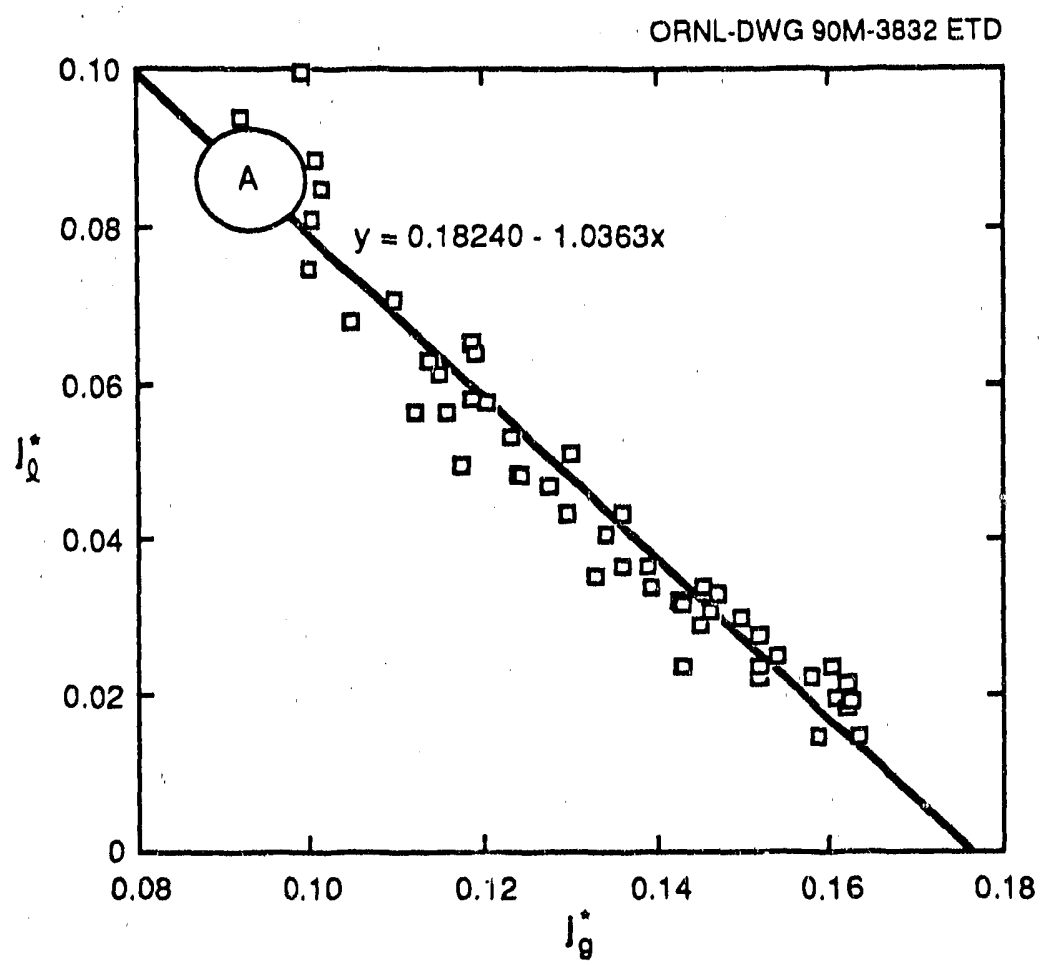

Fig. 16. HFIR Penetration Data

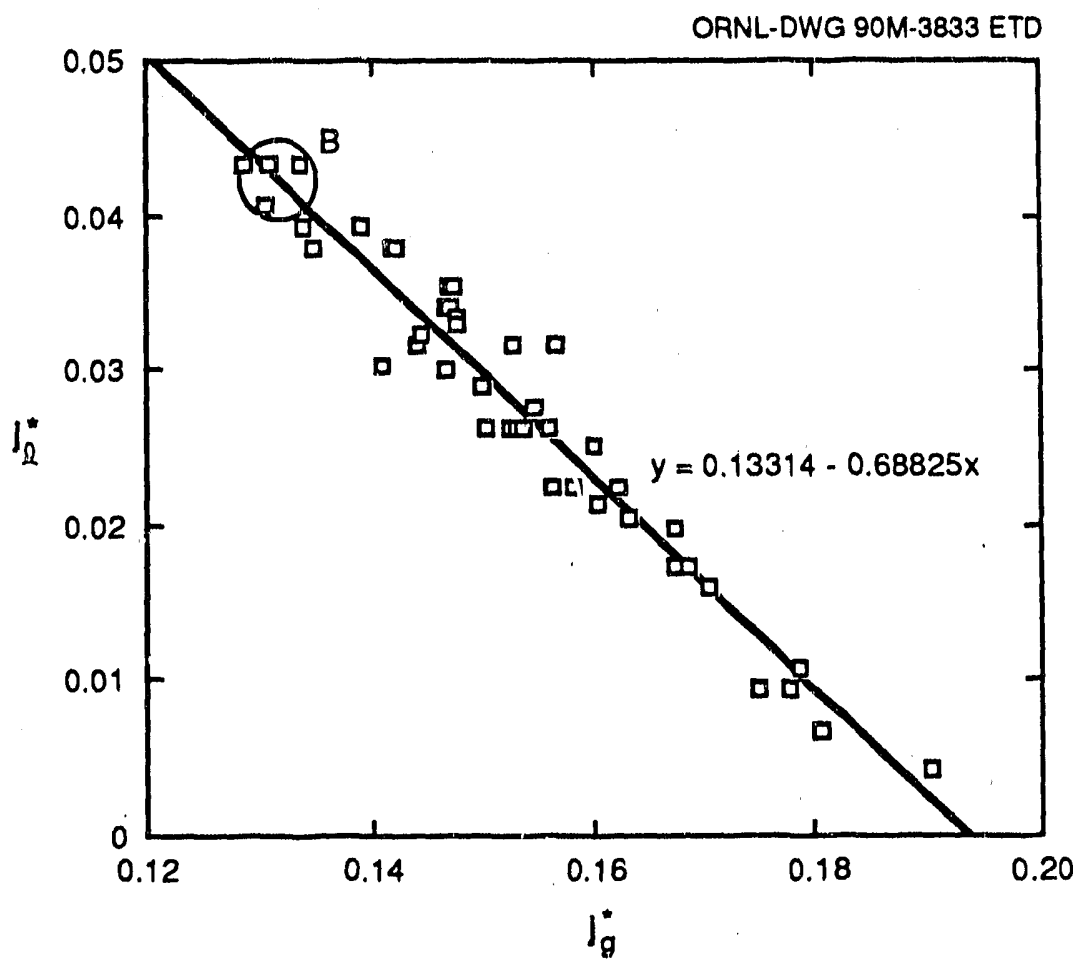

Fig. 17. HFIR Blow-out Data 
for the blowout characteristic. The data statistics associated with the blowout and penetration characteristics are given in Appendix $D$.

The CCF curve for a HFIR core channel can be calculated from the position of points $A$ and $B$ in Figs. 16 and 17, respectively, using Eq. (6) as,

$$
\left[j_{g}^{n}\right]^{1 / 2}+0.7\left[j_{i}^{\cdot 1 / 2}\right]^{1 / 2}=0.5
$$

This result is very close to the correlation proposed by Osakabe and Kawasaki, 1989, shown in Fig. 2 and given by

$$
\left[\dot{j}_{g}\right]^{1 / 2}+0.8\left[j_{i}^{\cdot}\right]^{1 / 2}=0.49
$$

\section{THE CCFL HEAT FLUX PREDICTED BY THE MEASURED CCFL DATA}

The CCFL heat flux for the HFIR fuel element is given by Eq. (9) as $12,000 \mathrm{w} / \mathrm{m}^{2}$, using the experimentally determined CCF performance given by Eq. (35). This corresponds tc a power level in the fueled region of the reactor of $0.62 \mathrm{Mw}$. It must be remembered that other factors external to the fuel element may cause more limiting heat transfer conditions. The calculations presented herein assume the availability of water both above and below the core.

\section{SUMMARY}

The CCF performance measured for a single channel in the parallel channel system used to cool the HFIR fuel element is consistent with that measured by Osakabe and Kawasaki, 1989, in single channel systems. Osakabe and Kawasali tested channel cross-sections with span-to-gap ratios varying from 10 to 50. The ratio of the span-to-gap in the HFIR is 66 . It appears that Eq. (9) is applicable for span-to-gap ratios within this range. However, infinite heat fluxes are predicted by Eq. (9) for channels of infinite span. This is not a physical limit since the channel gap will limit the amount of CCF. Therefore, the use of Eq. (9) is suspect for channels with cross sections far from those already evaluated experimentally.

The mechanistic model presented earlier can be applied to a HFIR channel. The CCF envelope predicted by the model can be approximated by

$j_{g}^{1 / 2}+0.57 j_{1}^{1 / 2}=0.63$.

This result is reasonably consistent with the HFIR test data as represented by Eq. (35). This result is also reason bly consistent with the correlation proposed by Osakabe and Kawasaki, 1989. 
The mechanistic model predicts a CCF envelope that is well represented by Eq. (1) with $m=0.55$ and $C_{w}=0.7$ when the span-to-gap ratio of the channel is 27 . Similarly, the mechanistic model predicts $m=0.57$ and $C_{w}=0.63$ when the span-to-gap ratio of the channel is 66 . Thus the performance of the mechanistic model is fairly insensitive to the span-to-gap ratio of the channel. This is consistent with the measured data and indirectly supports the assumption made in Eq. (23) to obtain the liquid fraction distribution in the channel. However, a careful study of the liquid distribution in the CCF of narrow channels is needed to validate the model.

Richter, 1981, and Pushkina and Sorokin, 1969, have shown that the CCF performance at liquid penetration is controlled by the Kutadeladze number when the characteristic dimension of the channel exceeds 40 times the dimension, $\lambda=\lambda_{T} / 2 \pi$, as defined in Fig. 3. Data in support of this were taken in tubes, annuli, and rod bundles. The value of the Kutadeladze number at liquid penetration is given as

$$
K u=\frac{\rho_{g}^{1 / 2} j_{\theta}}{\left[g \sigma\left(\rho_{1}-\rho_{g}\right)\right]^{1 / 4}}=3.2 \text {. }
$$

The value of $j_{g}$ at zero liquid penetration is thus independent of the channel dimension for large channels. The ciannel span in the HFIR fuel element is approximately 33 times the characteristic dimensiolı used for CCF in large tubes and rod bundles as defined in Fig. 3. Thus the CCF performance in narrow channels of large span may approach a limit similar to that for large lubes, annull, and rod bundles. However, a value for the Kutadeladze number of 9.6 would better represent the superficial vapor velocity at zero liquid velocity as predicted from Eq. (35).

The phase distribution across the channel span will cause variations in the local heat transfer conditions. This may allow CHF to be obtained at the span centerline prior to the CCF limit. Mishima and Nishihara, 1985, observed occasional dryout of the heated surface near the span centerline in their diabatic CCF experiments. A simple one dimensional conduction analysis is presented in Appendix $E$ that indicates a core power of $1 \mathrm{Mw}$, applied locally along the span centerlines will not result in core melting as long as the span ends remain wet. Thus local heat transfer coefficient variations across the span are not expected to cause premature CHF in the blocked downcomer situation.

\section{CONCLUSIONS}

The CCFL heat flux in the HFIR fuel element is calculated from experimentally determined CCF performance as $12,000 \mathrm{w} / \mathrm{m}^{2}$. The CCF performance was measured in a full-scale HFIR fuel element with careful consideration of parallel channel effects and channel inlet conditions. Thus the CCF data produced is applicable to the performance of the HFIR fuel element with minimal consideration of nonprototypic experimental conditions. The experimentally determined CCF performance is reasonably consistent with other data existing in the literature, despite the parallel channel configuration and the unusual channel cross section. 


\section{REFERENCES}

1. D. C. Baird, "Experimentation: An Introduction to Measurement and Theory and Experiment Design," Prentice-Hall, 1962.

2. S. G. Bankoff and S. C. Lee, "A Critical Review of the Flooding Literature," NUREG/CR. $3060,1983$.

3. S. G. Bankoff, "Minimum Thickness of a Draining Liquid Film," International Journal of Heat and Mass Transfer, Vol. 14, 2143-2146 (1971).

4. M. R. Fakory and R. T. Lahey, Jr., "Parallel Channel Effects and Long-Term Cooling During Ernergency Core Cooling in a BWR/4," NUREG/CR-3376, 1983.

5. W. J. Frea, "Two-Phase Heat Transfer and Flooding in Countercurrent Flow," proceedings of the 14th International Heat Transfer Conference held at Paris, Paper No. B5.10, 1970.

6. W. R. Gambill and R. D. Bundy, "Burnout Heat Fluxes for Low-Pressure Water in Natural Circulation," ORNL-3026, 1960.

7. P. Griffith, "The Prediction of Low-Quality Boiling Voids," Journal of Heat Transfer, 327-333, (1964).

8. P. Giiffith, W. A. Schumann, A. D. Neustal, "Flooding and Burn-out in Closed-end Vertical Tubes," Two-phase Fluld Flow Symposium, Paper No. 5, Institution of Mechanical Engineisrs, London, 1962.

9. V. lannello and N. E. Todreas, "Mixed Convection in Parallel Channels with Application to the ZLiquid-Metal Reactor Concept," Nuclear Sclence and Engineering, Vol. 101, 315-329, (1989).

10. E. Kreyszig, Advanced Engineering Mathematics, pub., John Wiley and Sons, Inc., 1972.

11. H. Kusuda and H. Imura, "Stability of a Liquid Film in a Countercurrent Annular TwoPhase Flow," Japan Society of Mechanical Engineers, Vol. 17, 1613-1618 (1971).

12. M. Ledinegg, "Instability of Flow During Natural and Forced Convection," Die Warm, Vol. 61. No. 8, 1938, AEC-TR-1861,(1954).

13. K. Mishima and M. Insii, "Critical Heat Flux Experiments Under Low Flow Conditions in a Vertical Annulus," NUREG/2647, ANL-82-6, 1982.

14. K. Mishima and H. Nishihara, "Effect of Channel Geometry on Critical Heat Flux for Low Pressure Water," International Journal of Heat and Mass Transfer, Vol. 30, No. 6, 1169-1182, (1987). 
15. K. Mishima, H. Nishihara, and I Michiyoshi, "Boiling Burnout and Flow Instabilities," International Journal of Heat and Mass Transter, Vol. 28, No. 6, 1115-1129 (1985).

16. K. Mishima and H. Nishihara, "The effect of Flow Directlon and Magnitude on CHF for Low Pressure Water in Thin Rectangular Channels," Nuclear Engineering and Design, Vol. 86, 165-181 (1985).

17. K. Mishima and $H$. Nishihara, "Flooding Velocitles for Countercurrent Air-Water Flow in Thin Rectangular Channels," Annual Report of the Reactor Institute of Kyoto University, Kyoto, Japan, Vol. 17, 1-14, 1984.

18. K. Mishima et al., "A Study of Alr-Water Flow in a Narrow Rectangular Duct Using Image Processing Technique," Japan-U.S. Seminar on Two-Phase Flow Dynamics, Ohtsu, Japan, July 1988.

19. K. Mishima and M. Ishil, "Critical Heat Flux Experiments Under Low-Flow Conditions in a Vertical Annulus," NUREG/CR-2647, ANL 82-6, 1986.

20. K. Ohkawa and R. T. Lahey, Jr., "The Analysis of CCFL Using Drift-Flux Models," Nuclear Engineering and Design, Vol. 61, 245-255, (1980).

21. M. Osakabe and Y. Kawasakl, "Top Flooding in Thin Rectangular and Arnular Passages," International Journal of Multiphase Flow, Vol. 15, No. 5, 747-754, (1989).

22. A. G. Ostrogorsky, R. R. Gay, and R. T. Lahey, Jr., "The Analysis of Countercurrent TwoPhase Flow Pressure Drop and CCFL Breakdown in Diabatic and Adiabatic Conduits," NUREG/CR-2386, 1981.

23. O. L. Pushkina and Y. L. Sorokin, "Breakdown of Liquid Film Motion in Vertical Tubes," Heat Transfer Soviet Research, Vol. 1, No. 5, 1969.

24. H. J. Richter, "Flooding in Tubes and Annull," International Journal of Multiphase Flow, Vol. Z, No. 6, 647-658, (1981).

25. H. Schlicting, Boundary-Layer Theory, MoGraw-Hill Book Company, 1979.

26. H. Suzukl, S. Hatamiya, and M. Murase, "Parallel Channel Effects Under BWR LOCA Conditions," Nuclear Engineering and Design, Vol. 109, No. 3, 399-405, (1988).

27. D. G. Thomas and S. K. Combs, "Measu' ement of Two-Phase Flow at the Core/Lipper Plenum Interface for a PWR Geometry Under Simulated Reflood Conditions," NUREG/CR3138, ORNL/TM-8204, 1983.

28. G. B. Wallis, One-dimensional Two-phase Flow, McGraw-Hill Book Company, 1969. 


\section{$31 / 32$}

29. G. B. Wallis et al., "Counterourrent Annular Flow Reglmes for Steam and Subcooled Water in a Vertical Tube," EPRI NP-1336, 1980.

30. G. B. Wallis of al., "Countercurrent Gas-Liquid Flow in Parallel Vertical Tubes," International Journal of Multiphase Flow, Vol. Z, 1-19, (1981).

31. N. Zuber, "Hydrodynamic Aspeots of Boilling Heat Transfer," AECU-4439, 1959. 
${ }_{33} / 34$

\section{APPENDIX A:}


Mechanistic CCF Model (Fortran Listing)

C VOID VS SUPERFICIAL VELOCITIES

CHARACTER" 1 TAB

OPEN (1,FILE-'SVGSVL')

GAP $=0.00127$

SPAN $=40 . *$ GAP

SPANR $=0.10$

$\mathrm{ROG}=1.18$

ROL $=1000$.

CLC $=0.008$

$C L W=0.008$

AXS-GAP*SPAN

$P=2$." (GAP+SPAN)

G $=9.8$

$A L=0.0$

DO $10 \mathrm{~J}=1,5$

$A L=A L+0.05$

$S V G=1$.

DO $10 \quad l=1,20$

$A G=1 .-A L$

TAUIG $=C L C / 2 . *(1.0+75 . " A L)$

TAUIS=CLC/2."(1.0+75."AL)

SVLSG=((AL"*2)/4.) "(TAUIG"ROG*(SVG/AG)"*2

1 "(4./(GAP*AL)+(1.-AL/2.)"P/(AG"AXS))

1 -(ROL-ROG)"G)"2."GAP"AL/4."CLW*ROL)

SVLSS=((AL"*2)/4.)"(TAUIS"ROG*(SVG/AG)"*2

1 "(4./(SPAN"AL)+(1.-AL/2.)"P/(AG"AXS))

1 -(ROL-ROG)"G)"2."SPAN"AL/4."CLW*ROL)

SVLSGS-SVLSG""2

SVLSSS-SVLSS"*2

SVLG=SVLSGS"*.25*(SVLSG/ABS(SVLSG))

SVLS=SVLSSS"*.25"(SVLSS/ABS(SVLSS))

SVLS=SVLS*GAP*25.SPANR

ALR=(SPAN**2"AL+AXS"AL/2.)/(SPANR*GAP)

SVL-SVLS-SVLG

DPI-((AG-1)"ROL-AG"ROG)"G+

1 ((CLW"ROL"4."(SVLS/AL)"*2/2.)

1 "2."GAP/AXS)+

2 ((CLW*ROL"4."(SVLG/AL)"**2/2.)

3 "2."SPAN/AXS)+

4 (TAUIG*ROG*(SVG/AG)"*2/2.)"P/(AG*AXS)

DPGAS-(TAUIG"ROG"(SVG/AG)"*2/2.)"(1.-AL/2.)"P/(AG"AXS)

SVLST=(SVL"(ROL/((ROL-ROG)*G"2."SPANR))**0.5) 


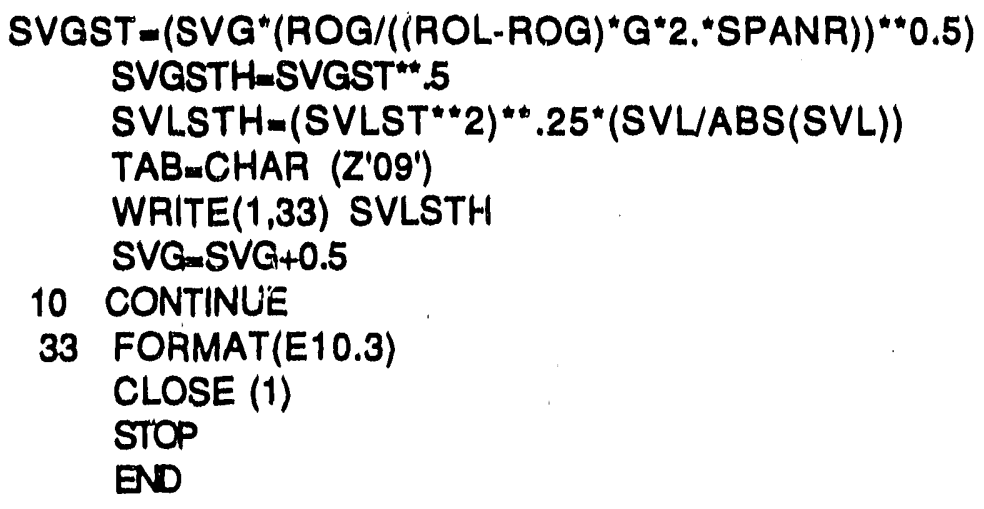


$37 / 38$

APPENDIX B: 


\section{Instrument Performance and Data Reduction Technique}

The air flow, water flow, and pressure measurement instruments were all calibrated using facilities in the Physical and Electrical Standards Laboratory. The calibration curves for the air measurement instruments are given in Figs. B1, B2, and B3. These meters were of the vortex shedding type. The water flow was measured using a rotameter. The calibration curve for the water-flow measurement instrument is given in Fig. B4. The pressure was measured at the vortex shedding meters and at the upper and lower plenums. The pressure measurement instruments were calibrated in place using a dead weight pressure tester. All instruments were operated within their linear ranges. Thermocouples were positioned at the vortex shedding meters to measure the temperature of the inlet air. The inlet water temperature was also measures. The inlet air was maintained $3^{\circ}-5^{\circ} \mathrm{C}$ above the inlet water temperature. The impact of humidification of the air and cooling of the air in the test section on the measured CCF performance was small, as indicated by Wallis et al., 1980.

The vortex shedding meter voltage output is proportional to the Strouhal number,

$\operatorname{Str}=\frac{\mathrm{nD}}{\mathrm{u}}$.

where $n$ is the vortex shedding frequency, $u$ is the velocity of the flow, and $D_{c}$ is some characteristic dimension (e.g., the diameter in the case of a cylindrical obstruction). The Strouhal number for the flow meters is independent of the local Reynolds number for the region of pressures and velocities measured in this experiment. Thus, the meters measure the local volume flux. The pressure at the test section is less than the pressure at the meter location. Thus the measured volume flux, $(d V) /(d t)_{\text {meas, }}$ must be increased to account for the air expansion. A constant temperature ideal gas process is assumed such that

$\frac{d V}{d t}_{\text {Actual }}=\frac{d V}{d t}\left[\frac{p_{\text {meas }}}{p_{\text {mactual }}}\right]$.

The actual test section pressure was taken as the average of the upper and lower plenum pressures.

Three Duroflow model 4512 Blowers provided the air flow to the test section. Each blower was fitted with a $100 \mathrm{Hp}$ induction motor. The blowers are of a semi-positive displacement type and very overpowered for this application. The inlet aiflow varied less than $5 \%$ as a result of the head variations the blowers incurred during these tests, as calculated from the performance data supplied by the manufacturer. 


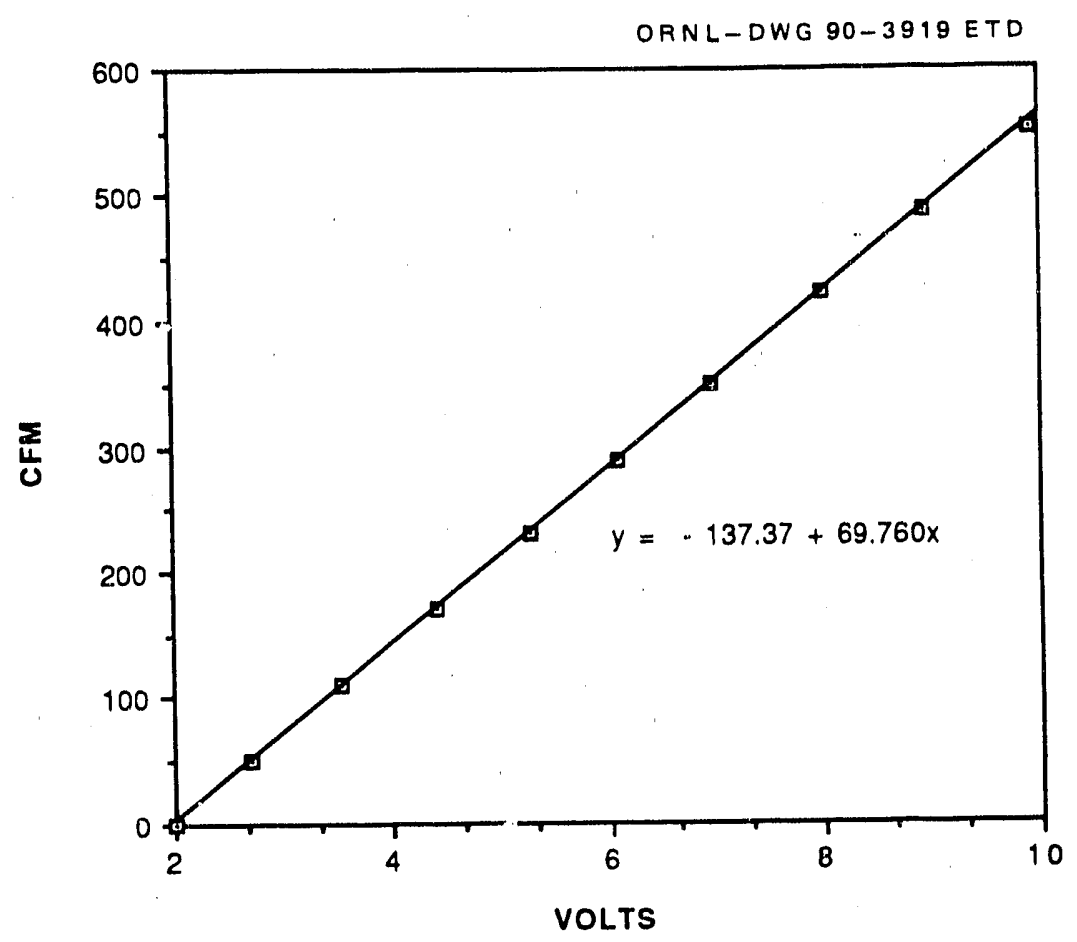

Fig. B1. Calibration of Vortex Shedding Meter \#1.

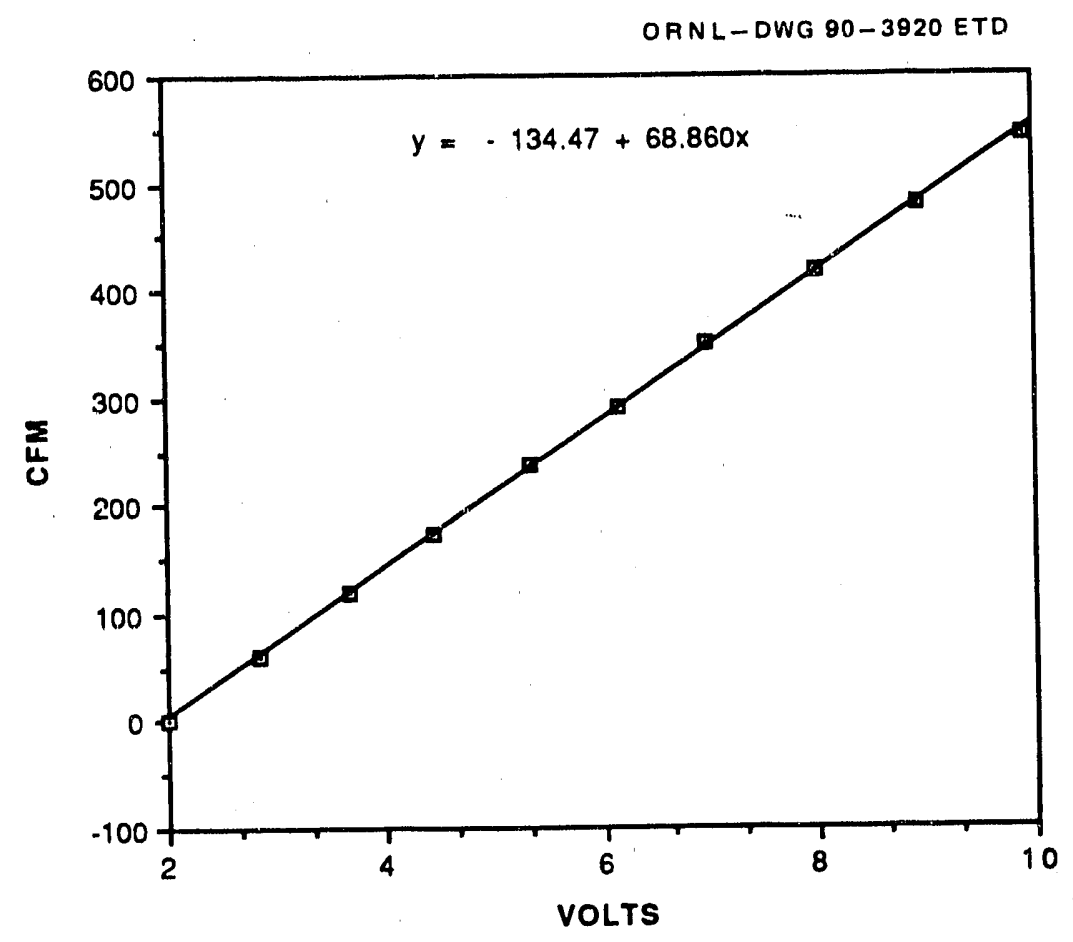

Fig. B2. Calibration of Vortex Shedding Meter \#2. 


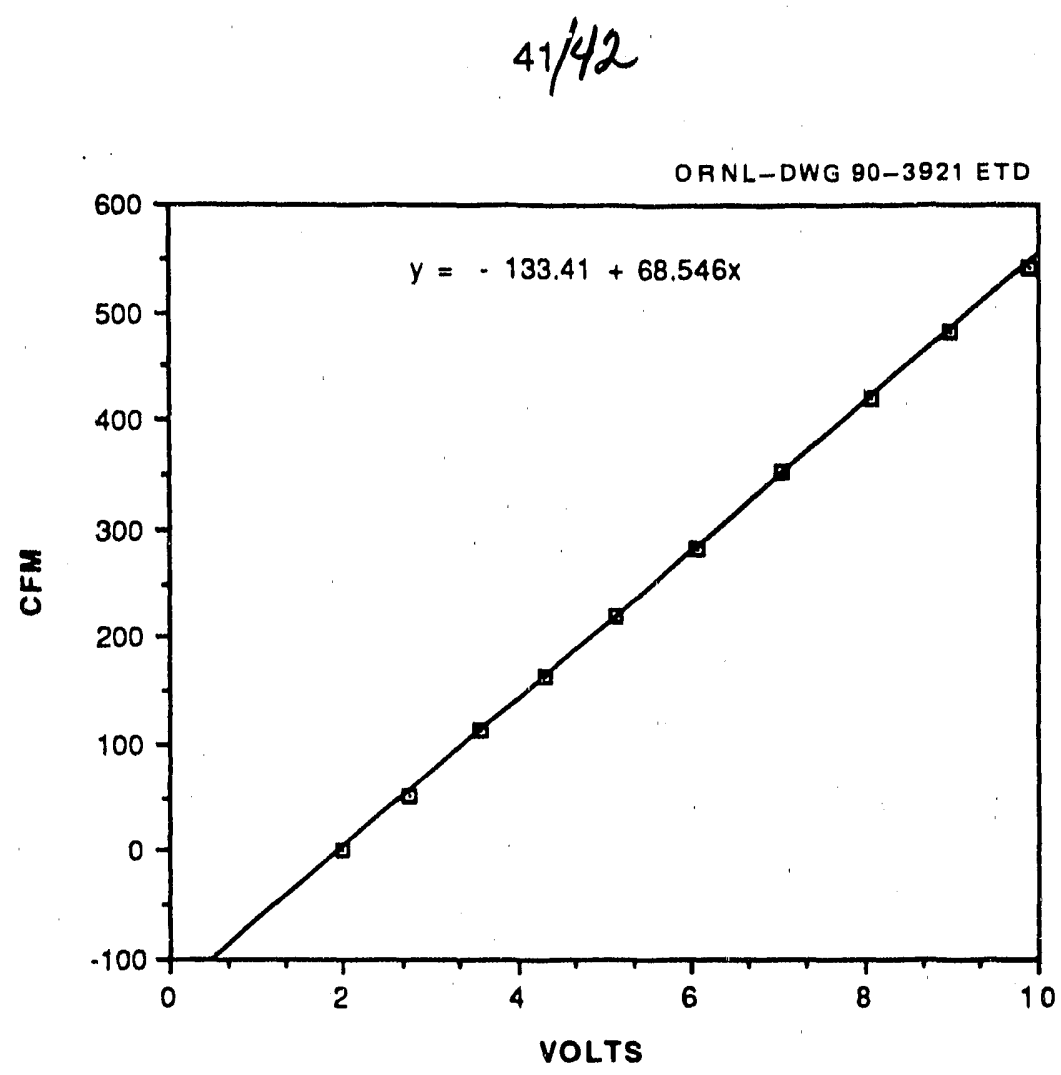

Fig. B3. Calibration of Vortex Shedding Meter \#3.

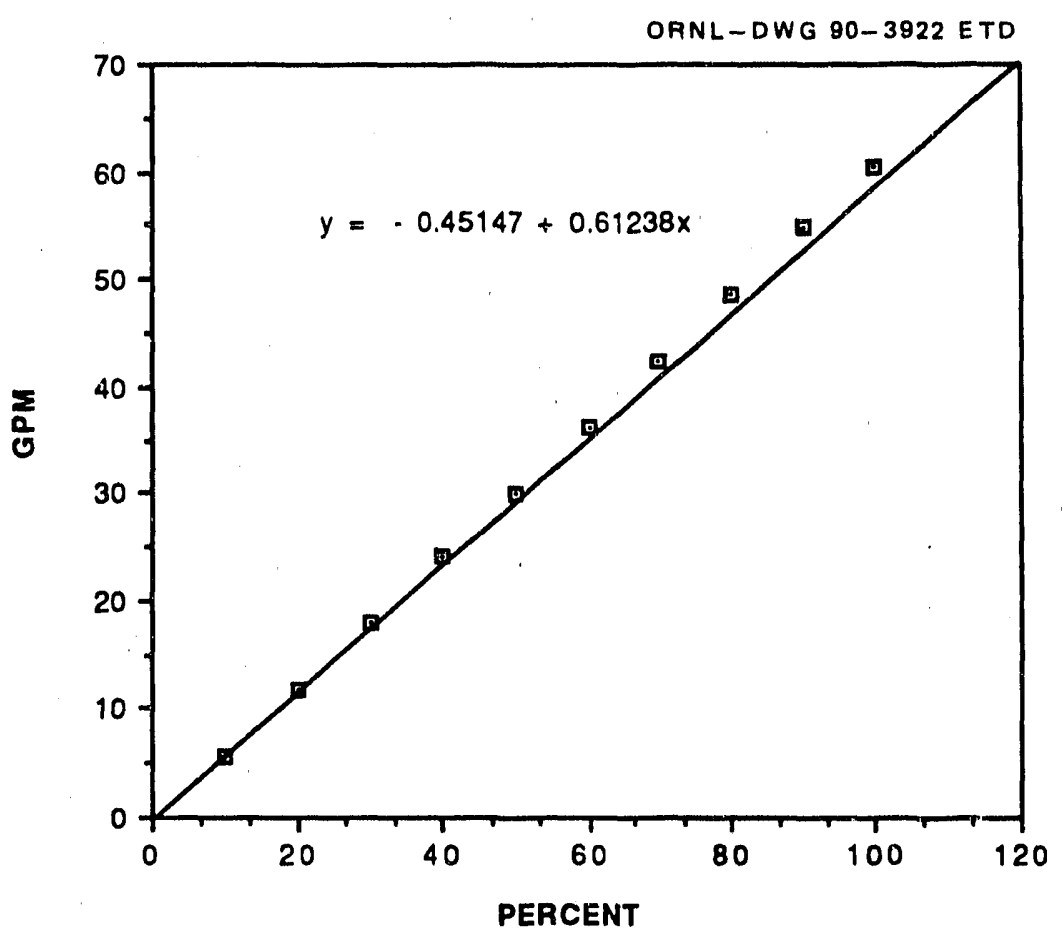

Fig. B4. Rotameter Calibration Curve. 
$43 / 44$

APPENDIX C: 
Table C.1 Penetration and Blowout Data

\section{PENETRATION}

$$
\left(j_{g}\right)^{*}
$$

0.16353059884

0.1609013931

0.15178414734

0.14509616772

0.13944787402

0.133959532

0.12939091214

0.117464515

0.16231096728

0.1472555154

0.13611887968

0.13014068564

0.15823219944

0.15198408694

0.1588520122

0.142956814

0.12423247046

0.14257692876

0.13301981588

0.12392256408

0.16249091292

0.1387580824

0.122962854

0.1620510458

0.15211404768

0.1452561194

0.1605514988

0.15413343764

0.14973476644

0.14621582948

0.1428568442

0.13608888874

0.1275114799 (j) *

0.0146349

0.0197253

0.0224826

0.0288456

0.0341481

0.0405111

0.0432684

0.0496314

0.02121

0.0328755

0.0432684

0.050904

0.0224826

0.0237552

0.0146349

0.0237552

0.0483588

0.0322392

0.0354207

0.0483588

0.019099

0.0366333

0.053025

0.0184527

0.027573

0.0341481

0.0237552

0.0250278

0.0301182

0.0307545

0.0316029

0.0366933

0.0470862

\section{BLOWOUT}

\begin{tabular}{ll}
\multicolumn{1}{c}{$\left(j_{g}\right)^{*}$} & $\left(j_{1}\right)^{*}$ \\
0.1599016951 & 0.0250278 \\
0.15639275512 & 0.0316029 \\
0.1443563912 & 0.0316029 \\
0.1468556362 & 0.0301182 \\
0.1620510458 & 0.02239776 \\
0.1671495056 & 0.0171801 \\
0.1777463044 & 0.0093324 \\
0.12882108428 & 0.0432684 \\
0.13409948972 & 0.0392385 \\
0.14192712506 & 0.0379659 \\
0.14744545802 & 0.0354207 \\
0.15288381514 & 0.0316029 \\
0.1545533108 & 0.027573 \\
0.14443636704 & 0.0322392 \\
0.13078049236 & 0.0405111 \\
0.13523914544 & 0.0379659 \\
0.14116735458 & 0.03020304 \\
0.15601286988 & 0.0263004 \\
0.1902425294 & 0.004242 \\
0.14714554862 & 0.0341481 \\
0.15297378796 & 0.0263004 \\
0.17481718926 & 0.0093324 \\
0.1684990979 & 0.0171801 \\
0.16037155316 & 0.02121 \\
0.15632277626 & 0.0224826 \\
0.15037457316 & 0.0263004 \\
0.14759541272 & 0.0328755 \\
0.13912797066 & 0.0392385 \\
0.13392954106 & 0.0432684 \\
0.18057544974 & 0.0067872 \\
0.1785960477 & 0.0108171 \\
0.17025856638 & 0.0159075 \\
0.16725947238 & 0.0197253 \\
0.16311072568 & 0.0203616 \\
0.15802226286 & 0.0224826 \\
0.1525539148 & 0.0263004 \\
0.15006466678 & 0.0288456 \\
0.14756542178 & 0.0335118 \\
0.14701558788 & 0.0354207 \\
0.15383352824 & 0.0263004 \\
0.14692561506 & 0.0341481 \\
0.14222703446 & 0.0379659 \\
0.130960438 & 0.0432684
\end{tabular}

0.0250278

0.0316029

0.0301182

0.02239776

0.0432684

0.0392385

0.0379659

0.0354207

(

0.0322392

0.0405111

0.03020304

0.0263004

0.004242

0.0341481

0.0093324

0.0171801

0.02121

0.0328755

0.0392385

0.0432684

0.0067872

0.0159075

0.0197253

0.0203616

0.0263004

0.0288456

0.0335118

0.0354207

0.034148

0.0432684 
$47 / 48$

!

APPENDIX D: 


\section{CCF Data Statistics}

The HFIR CCF data associated with the penetration and blowout characteristics were analyzed using the Statistical Analysis System (SAS), 1982 edition. This is a commercial software package produced by SAS Institute, Inc. This package was used to generate the least squares linear fit to the blowout and penetration data. Standard deviation of the slope and intercept values associated with each linear fit are given in Table D1.

Table D.1 Statistics of the Linear Least Squares Fit to the Data

\begin{tabular}{lllll} 
& \multicolumn{2}{c}{ Intercept (CEM) } & \multicolumn{2}{c}{ Slepe (CEM/GPM) } \\
Penetration Line & $b_{p=0.182}$ & $(\sigma=0.002)$ & $m_{p}=1.04$ & $(\sigma=0.04)$ \\
Blowout Line & $b_{b}=0.133$ & $(\sigma=0.001)$ & $m_{b}=0.69 \quad(\sigma=0.03)$
\end{tabular}

The standard deviation of the slope and intercept values of each line are not independent. Uncertainty of the order of plus or minus 0.05 exists in the nondimensional superficial gas velocity corresponding to the departure from constant pressure which locates point $A$ along the penetration line. Likewise, uncertainty of plus or minus 0.05 exists in the nondimensional superficial gas velocity which corresponds to the position of point $B$ along the blowout line. The CCF performance of the HFIR fuel assembly is calculated from the position of points $A$ and $B$ as shown graphically in Fig. D1. Note that both the penetration and blowout curves are nearly tangent to the predicted CCF performance at points $A$ and $B$, respectively. Therefore, the calculated CCF curve, Eq. (35), is not very sensitive to uncertainty in the position of points $A$ and $B$ along the penetration and blowout lines. The uncertainty associated with the coefficients $m$ and $\mathrm{C}_{w}$ are primarily associated with the uncertainty in the slope of the penetration and blowout curves. These slope values are used to calculate the superficial liquid velocities that correspond to the superficial points $A$ and $B$ along the penetration and blowout curves. The CCFL heat flux given by Eq. (9) goes as the coefficient $C_{w}$ squared, and is not very sensitive to the coefficient $m$. Therefore, the limiting values for the CCFL heat flux are established by the limiting values in $C_{w}$. The lower value for $C_{w}$ in Eq. (35) is established by choosing the maximum superficial liquid velocity value corresponding to point $A$ (i.e., calculated using the mean slope plus two standard deviations for the penetration line) and the minimum superficial liquid velocity corresponding to point B (i.e., calculated using the mean slope minus two standard deviations for the blowout line). The variation in the intercept values has negligible impact on the predicted values of $m$ and $C_{w}$. Conversely, the upper value for $C_{w}$ is calculated using the minimum superficial velocity value for point $A$ and the maximum superficial velocity value for point $B$.

The lower limit values for $m$ and $C_{w}$ are 0.48 and 0.45 , respectively. The upper limit values for $m$ and $C_{w}$ are 0.98 and 0.57 , respectively. The $95 \%$ confidence interval limits for the CCFL core power are from $0.53 \mathrm{Mw}$ to $0.73 \mathrm{Mw}$ with the mean value correspo. uding to $0.62 \mathrm{Mw}$. 
$51 / 52$

\section{APPENDIX E:}




\section{HFIR Fuel Plate Scoping Conduction Analysis}

The phase distribution across the span of a narrow rectangular channel causes corresponding variations in the local heat transfer coefficient. A first-order analysis is presented herein to establish heat transfer limits associated with conduotlon along the channel span. It is assumed that all the core power is deposited along the centerline of the fuel-plate span. It is further assumed that the span ends remain wet. Therefore, the temperature at the span edge is taken as the saturation temperature at the associated pressure. The model for this case is shown in Fig. E-1.

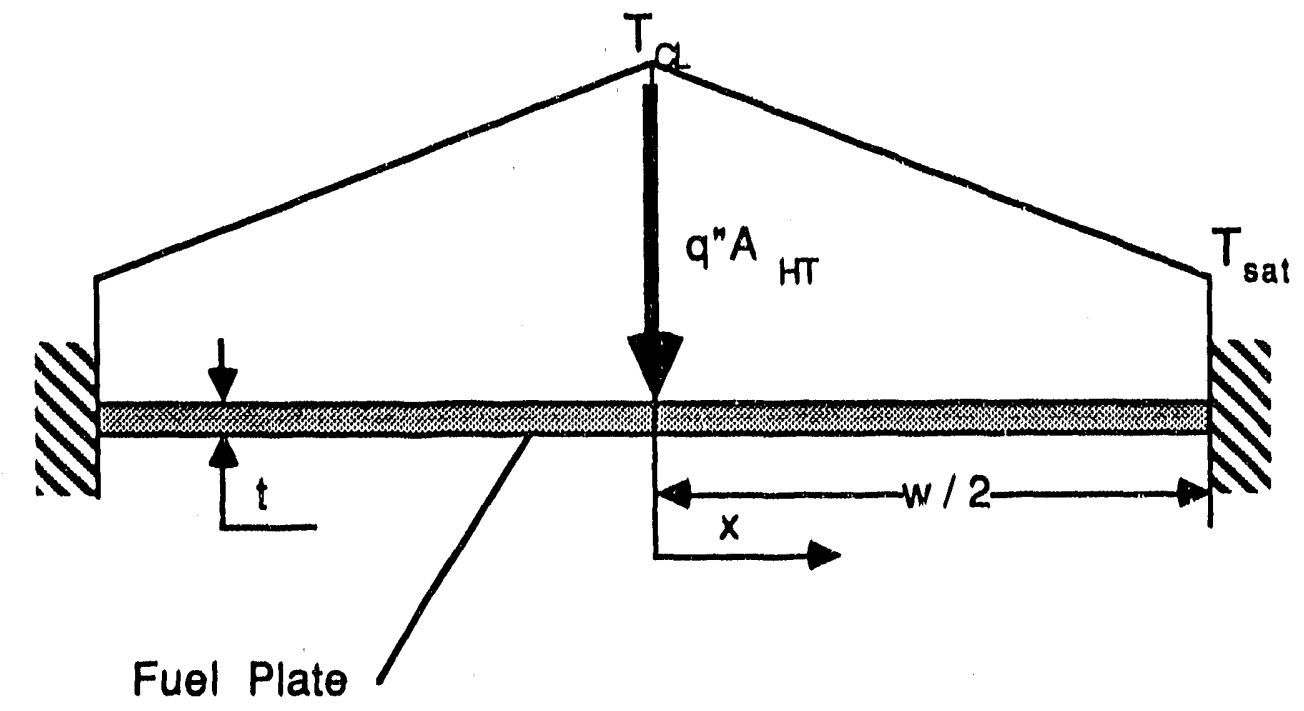

Fig. E.1 Fuel Plate and Associated Boundary Conditions for First Order Conduction Analysis

The fuel plate span centerline temperature can be expressed in terms of the plate geometry and associated material properties using a one-dimensional conduction model,

$T_{a}=T_{\text {sat }}+\frac{w^{2} q^{\prime \prime} \text { ore }}{2 k t}$

HFIR fuel plate dimensions and water properties at $0.15 \mathrm{MPa}$ give a case specific value for the centerline temperature of $475^{\circ} \mathrm{C}$ when the core heat flux is $25,000 \mathrm{~W} / \mathrm{m}^{2}$. This corresponds to a core decay power of $1.2 \mathrm{MW}$. This analysis is intended to provide intuition concerning the importance of conduction heat transfer in circumstances of decay heat removal. A more exacting and detailed conduction analysis would be warranted if this analysis hinted of potential problems due to temperature gradients across the span of the fuel plates. 

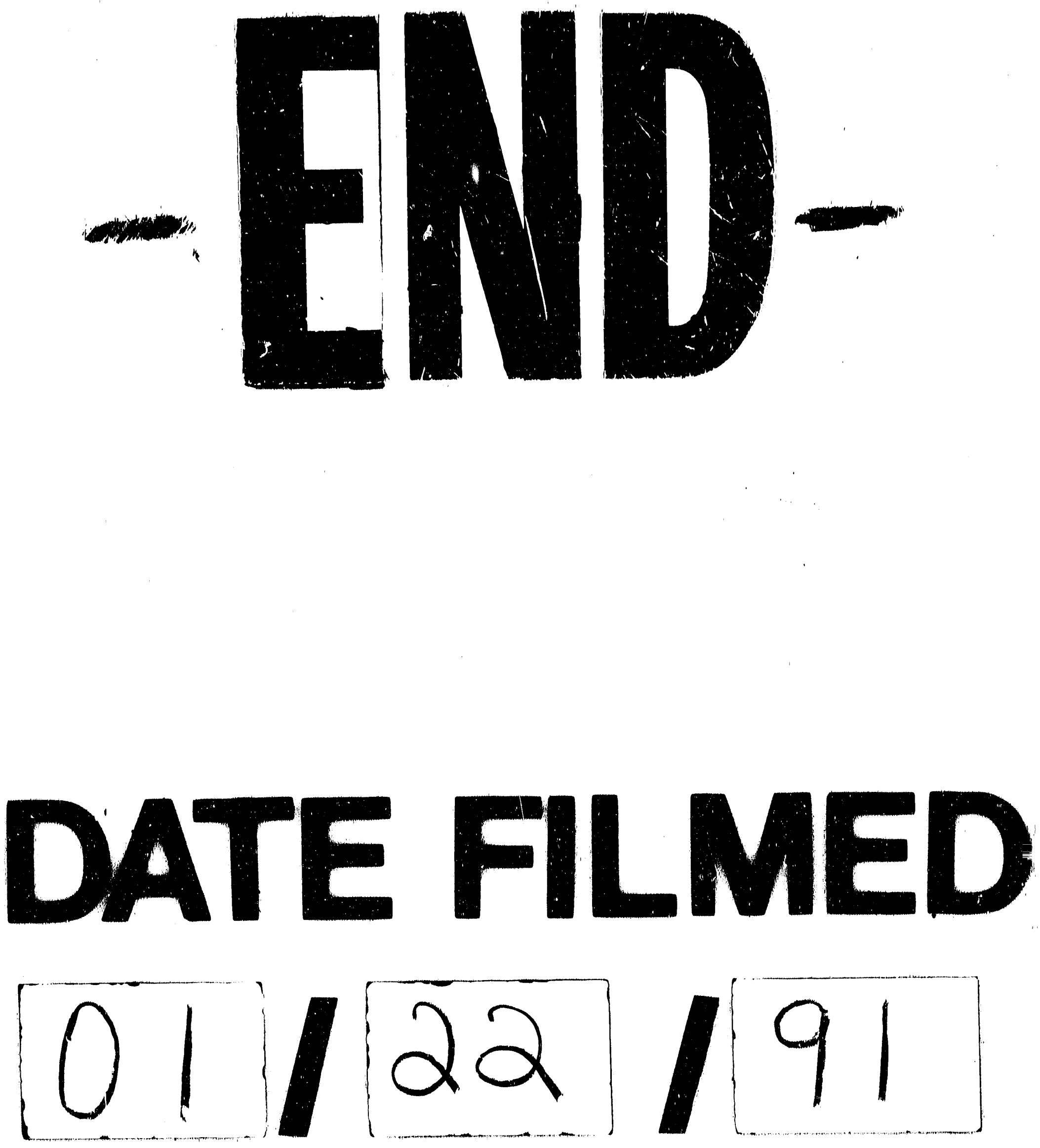
